\title{
Boundary layer transition over a foil using direct numerical simulation and large eddy simulation
}

Cite as: Phys. Fluids 31, 124102 (2019); https://doi.org/10.1063/1.5126663

Submitted: 04 September 2019 . Accepted: 05 November 2019 . Published Online: 04 December 2019

T. A. Smith (D), and Y. Ventikos (D)

\section{ARTICLES YOU MAY BE INTERESTED IN}

Flow structures in transitional and turbulent boundary layers

Physics of Fluids 31, 111301 (2019); https://doi.org/10.1063/1.5121810

Large scale instabilities in coaxial air-water jets with annular air swirl

Physics of Fluids 31, 124103 (2019); https://doi.org/10.1063/1.5122273

Effects of upstream perturbations on the solution of the laminar and fully turbulent boundary layer equations with pressure gradients

Physics of Fluids 31, 125103 (2019); https://doi.org/10.1063/1.5125496

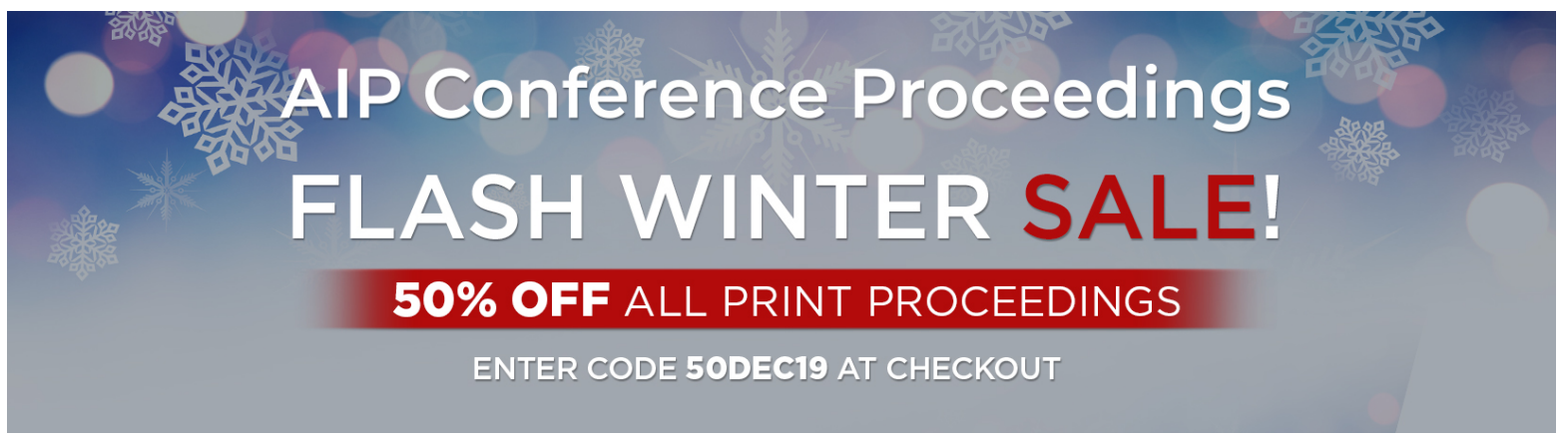




\title{
Boundary layer transition over a foil using direct numerical simulation and large eddy simulation
}

\author{
Cite as: Phys. Fluids 31, 124102 (2019); doi: 10.1063/1.5126663 \\ Submitted: 4 September 2019 - Accepted: 5 November 2019 • \\ Published Online: 4 December 2019
}

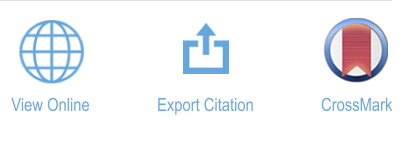

\author{
T. A. Smith ${ }^{a)}$ (D) and Y. Ventikos
}

\author{
AFFILIATIONS \\ Department of Mechanical Engineering, University College London, London WC1E 7JE, United Kingdom \\ a) Electronic mail: tom.smith.17@ucl.ac.uk \\ ${ }^{b)}$ Electronic mail: $y$.ventikos@ucl.ac.uk
}

\begin{abstract}
Transitional boundary layers over lifting bodies represent an important class of flows in many industrial applications, and accurately capturing the transition is crucial for the prediction of important phenomena such as lift, drag, and trailing-edge noise. In this study, we consider how large eddy simulations (LESs) can be used to capture the natural boundary layer transition and compare the results to fully resolved direct numerical simulations that provide a detailed picture of the transition and trailing edge flow. The ability of LES to capture the transition is considered by looking at different elements of the subfilter scale modeling and discretization. The behavior of the subfilter scale model is shown to be critical, and it must remain inactive during the early stages of transition to avoid erroneous predictions due to excessive dissipation. Dispersion errors, when present, can cause the natural transition mechanism to be bypassed at an earlier stage, which leads to higher levels of turbulent kinetic energy at the trailing edge.
\end{abstract}

Published under license by AIP Publishing. https://doi.org/10.1063/1.5126663

\section{INTRODUCTION}

Transitional boundary layers are present in a wide range of flows in aerospace, marine, and a variety of other applications. For flows at moderate Reynolds numbers, the exact nature and location of the transition is important as this plays a crucial role in determining characteristics such as frictional drag. The dynamics of the boundary layer also influence the behavior of the trailing edge flow and tip-vortex formation, which are important acoustic sources in many applications. The frequency content of the fluctuations at the trailing edge is closely correlated with the resulting acoustic spectrum, and so accurate acoustic analyses are predicated on being able to predict the development and behavior of the boundary layer.

A large number of experimental and numerical studies have been carried out on boundary layer flows over flat plates and foil bodies in order to better understand the different mechanisms by which transition can occur. ${ }^{1}$ Particular attention has been paid to the transition of flat-plate boundary layers via the growth of Tollmien-Schlichting waves. ${ }^{2}$ The influence of separation bubbles on boundary layer transition, which are of particular importance to lifting surfaces, has also been explored in a number of works using a direct numerical simulation. ${ }^{3-5}$ Such efforts have resulted in a much improved understanding of the stages of transition, although some questions remain, particularly regarding the later nonlinear stages. The transition in a detached flow over a foil at low Reynolds numbers is considered in Ref. 6, which shows KelvinHelmholtz instabilities in the large separated region on the suction side. Experimental studies ${ }^{7,8}$ have also investigated the dynamics of laminar separation bubbles and their role in boundary layer transition. These studies have provided detailed insights into the complex interactions between the separation and transition points as well as effects of the trailing edge flow on the upstream bubble. Studies such as these highlight the challenging flow physics that numerical models must be able to capture in order to correctly predict the dynamics of the boundary layer and trailing edge flow. Direct numerical simulations have also provided valuable insights into the effects of freestream turbulence on boundary layer transition. 90 The receptivity of the boundary layer to different intensities and scales of turbulence has been explored, ${ }^{9}$ showing how larger turbulent structures can bring forward the transition point through a mechanism known as bypass transition. 'While these studies have provided valuable insights into the detailed physics of boundary layer flows, DNS remains prohibitively expensive for most 
industrially relevant configurations and so one must look to alternative methods.

Large eddy simulation is based on the concept of filtering out the smallest turbulent scales and modeling their effect on the flow field separately. For a wide variety of flows, a large eddy simulation (LES) has been shown to be an effective way of drastically reducing the computational requirements compared to a DNS, while retaining the important flow features. However, one of the fundamental difficulties in using the LES for transitional boundary layers lies in resolving the scales that are responsible for the initial transition. This is known as the receptive stage, where disturbances in the flow field or unstable shear layers result in growing velocity fluctuations in the boundary layer. The use of large eddy simulations to model the transition process for a flat-plate boundary layer has been reported in a number of studies, for example, Refs. 11-14. In one study, ${ }^{12}$ large eddy simulations of the flow over a flat plate were considered to assess how well the skin friction was predicted at each stage. A number of different subfilter scale (SFS) models were considered, with the constant coefficient models proving to be overly dissipative and incapable of capturing the transition. The excessive damping is noted in other studies, for example. ${ }^{11}$ In another recent study, ${ }^{13}$ a forcing function was proposed based on linear stability theory to provide the initial disturbance for the transition of a flatplate boundary layer in order to overcome the high mesh resolutions required. Transition in a separated flow has also been considered. ${ }^{1.5}$ In this study, experimental data were used to provide a pressure field representative of a foil-type body that is then imposed on a flat plate.

An important application of modeling transitional boundary layer flows is the prediction of trailing edge noise. The relationship between boundary layer instabilities and trailing edge noise has been explored experimentally ${ }^{16,17}$ and also numerically ${ }^{18}$ using the direct numerical simulation. The focus of these studies has been on tonal noise induced by Tollmien-Schlichting waves that are amplified in a separated region close to the trailing edge on the pressure side of a foil. A number of criteria have been identified that, if satisfied, give rise to large amplitude tonal noise. One such requirement is that the boundary layer is still transitional at the trailing edge and hence correctly capturing the location and nature of the transition is vital for predicting such phenomena.

The use of large eddy simulations for the prediction of trailing edge noise has also been investigated in a number of recent works. A common approach, often referred to as a hybrid method, is to use the pressure and velocity fields from an incompressible LES as the input data for an acoustic model such as those proposed by Curle ${ }^{19}$ or Ffowcs-Williams and Hawkings. ${ }^{20}$ This approach is adopted in a number of works, for example, Refs. 21 and 22, among others. However, such studies tend to rely heavily on detailed experimental data for validation, and a lack of thorough verification of the simulations makes it difficult to think of LES as a predictive tool in this regard at present. Such simulations are still limited to moderate Reynolds numbers, with mesh resolutions often being commensurate with a "coarse" DNS in the near-wall region.

There is little consensus in the published literature of how best to carry out large eddy simulations for boundary layer flows, and a number of different subfilter scale (SFS) models and discretization approaches are used. While it is well known that the use of upwind-biased schemes introduces artificial dissipation into the solution, they are still widely used. This is primarily because pure central schemes can introduce numerical instabilities, which, if large enough, prevent a solution from being obtained. This is particularly true when higher Reynolds numbers considered as fine enough meshes are often not computationally feasible. In Ref. 22, the QUICK (quadratic upstream interpolation for convective kinematics) scheme is used for the convective term, whereas a bounded central scheme is adopted in Refs. 21 and 23. This is a type of blended scheme where a level of up-winding is used to stabilize the solution. A different study ${ }^{24}$ uses a fourth-order central scheme to minimize the dissipative effects of the discretization scheme. Numerous different subfilter scale models are also used. For example, the authors of Ref. 23 used the standard Smagorinsky model to look at the effects of biomimetic structures on trailing edge noise reduction. Dynamic models are also employed, most commonly the dynamic Smagorinsky model, for example, in Ref. 22. Detailed studies of the validity of the different approaches are scarce, and so further research is needed to improve confidence in the predictive capabilities of LES for phenomena such as trailing edge noise. A comparison of the performance of two different SFS models (wall-adapting and QR eddy-viscosity models) is considered in Ref. 25. Here, the performance is also assessed by considering the transitional flow over a foil. It is found that both models capture the transition process but with varying levels of accuracy. However, the sensitivity of the results to the mesh resolution and discretization scheme is not considered in this study.

One of the main challenges for using the large eddy simulation in this regard lies in the interaction between the numerical errors and the subfilter scale modeling. Depending on the modeling approach used, errors in the cut-off region can contaminate lower wavenumbers. A number of efforts to understand and quantify the different types of errors have been made for a range of different flows, for example, Refs. 26-29, but how well such methods apply to transitional boundary layers is less understood. It is clear from these works that there is a complex relationship between the grid resolution, the subfilter scale model, and the discretization scheme. A better understanding of this relationship when considering transitional boundary layer flows is therefore needed in order that appropriate methods and grids are used.

In this study, we consider the transition of the boundary layer over a foil (see Table I) at a Reynolds number of $R e=10^{5}$ with zero free-stream turbulence. The foil has a $4^{\circ}$ angle of attack which gives rise to a small, thin laminar separation bubble on the suction side. This results in an inviscid Kelvin-Helmholtz instability, which eventually leads to a fully developed turbulent boundary layer. The aim of this paper is to assess how large eddy simulations can be used to capture the natural transition of the boundary layer and the

TABLE I. Principle parameters and operating conditions.

\begin{tabular}{lc}
\hline \multicolumn{1}{c}{ Geometry } & NACA0012 \\
\hline Reynolds number (Re) & $1.0 \times 10^{5}$ \\
Angle of attack & $4.0^{\circ}$ \\
Freestream velocity & $5.0 \mathrm{~ms}^{-1}$ \\
Chord length & $0.3 \mathrm{~m}$ \\
Span & $0.06 \mathrm{~m}$ \\
\hline
\end{tabular}


resulting trailing edge flow. The analysis focuses on the transition process in order to better understand how the LES can be used to accurately model practical configurations involving transitional and turbulent boundary layers. The Kelvin-Helmholtz instability is also an important mechanism for the generation of turbulence in tipvortex flows, ${ }^{30,31}$ and so an understanding of how numerical methods capture this process is crucial to a wide range of applications pertaining to lifting surface flows, such as aircraft wing tips and marine propeller blades.

In this paper, fully resolved direct numerical simulations are carried out, which provides a detailed description of the separation bubble, boundary layer transition, and the subsequent spectral content of the fluctuations in the boundary layer. These data are used to validate a number of different large eddy simulations, where the effects of the subfilter scale model, discretization scheme, and mesh resolution are considered. The performance of the different modeling techniques is assessed in terms of the presence of different types of errors, including artificial dissipation and dispersion errors, with the DNS data providing an excellent benchmark that allows for such errors to be identified and understood. A key aim is to understand the limits of different modeling techniques with respect to the mesh resolution. The cost of DNS is prohibitive for many practical applications, whereas the LES can be applied more widely. In order for the large eddy simulation to be used with more complex geometries and higher Reynolds numbers, a better understanding of the mesh requirements and how to control different sources of error is needed.

For the LES, we consider several second-order discretization schemes with fixed amounts of blending. This allows us to understand the trade-off between instabilities, dispersion errors, and the effects of artificial dissipation at different points in the flow field. There are, of course, a number of schemes that use limiters or NVD (normalized variable diagram) approaches to do this. ${ }^{32-34}$ Such approaches use different measures of unboundedness to determine the required level of blending, and some, for example, the bounded central scheme used in Ref. 21, reduce to first-order in certain circumstances, which is highly undesirable for the LES. Because the level of blending varies across the flow domain when this approach is used, it can be difficult to understand the effect on the flow field. By fixing the level of blending, a clearer insight into the effects can be obtained.

Section II details the numerical approach and methods adopted in the study and the geometry; meshing and boundary conditions are discussed in Sec. III. The DNS results describing the flow field are provided in Sec. IV and results from the large eddy simulations are presented in Sec. $\mathrm{V}$, together with a discussion on the relative performance of the different methods. A number of conclusions are given in Sec. VI, along with recommendations on the verification for large eddy simulations of boundary layer flows.

\section{METHODS}

\section{A. Governing equations}

The governing equations for an incompressible Newtonian fluid are given in the following equations:

$$
\frac{\partial U_{i}}{\partial x_{i}}=0
$$

$$
\frac{\partial U_{j}}{\partial t}+U_{i} \frac{\partial U_{j}}{\partial x_{i}}=-\frac{1}{\rho} \frac{\partial p}{\partial x_{j}}+v \frac{\partial^{2} U_{j}}{\partial x_{i}^{2}} .
$$

For direct numerical simulations, all of the flow scales are resolved and so no further modeling is required. For the large eddy simulation, the flow field is split into a resolved part and a modeled part by spatially averaging the continuity and momentum equations. ${ }^{35}$ The latter part, representing the higher wave-number turbulence, is then modeled using a subfilter scale model. The filtered equations are derived by introducing a general filtering operator, $G$, to each variable,

$$
\bar{\phi}(\mathbf{x}, t)=\int_{V} G(\mathbf{r}, \mathbf{x}) \phi(\mathbf{x}-\mathbf{r}, t) d \mathbf{r} .
$$

Applying the filter to each of the variables, the filtered equations are obtained,

$$
\frac{\partial \bar{U}_{j}}{\partial t}+U_{j} \frac{\partial \bar{U}_{i}}{\partial x_{i}}=-\frac{1}{\rho} \frac{\partial \bar{p}}{\partial x_{j}}+v \frac{\partial^{2} \bar{U}_{j}}{\partial x_{i} \partial x_{i}}-\frac{\partial \tau_{i j}}{\partial x_{i}}
$$

where

$$
\tau_{i j}=\overline{U_{i} U_{j}}-\bar{U}_{i} \bar{U}_{j} .
$$

It is important to note that in the derivation of Eq. (4), it is assumed that the operations of filtering and differentiation commute. For this to be true in the general sense, the filter function must be spatially homogeneous. ${ }^{36}$ As will be shown later, the filter width is usually taken to be some function of the grid size and so will not, in general, satisfy this condition. However, the error will remain small so long as the gradients in the mesh remain small. This is an important consideration when designing meshes for the LES.

\section{B. Large eddy simulation}

The filtered equations given by Eq. (4) are not closed, and so we must introduce an additional model to account for the effects of the subfilter scale (SFS) stresses, $\tau_{i j}$. In this study, two different models for the subfilter scale stresses are considered: the Smagorinsky model and the local dynamic $\mathrm{k}$ model.

The Smagorinsky model ${ }^{37}$ is widely used and is an eddy viscosity model based on a mixing length, which is usually defined by the grid size. The eddy viscosity assumption relates the SFS viscosity to the stress tensor through

$$
\tau_{i j}=-2 v_{S F S} \overline{S_{i j}},
$$

where $\bar{S}_{i j}$ denotes the filtered rate-of-strain. The subfilter scale viscosity is determined using a lengthscale which is related to the filter width through a constant, $C_{s}$, known as the Smagorinsky constant,

$$
v_{S F S}=C_{s} \Delta \bar{S} .
$$

The Smagorinsky constant can vary considerably from one flow to the next, but a value of 0.1 is often used and this is adopted here.

From fundamental boundary layer theory, it is known that turbulence must vanish at the wall. Looking at the Smagorinsky model, one can see that there is no provision for this and so it is necessary to employ a damping function to force $v_{S F S}=0$ at the wall. Moin and $\mathrm{Kim}^{38}$ proposed using a van Driest damping function ${ }^{39}$ which is an 
empirical function based on the law-of-the-wall. The lengthscale in Eq. (7) then becomes

$$
l_{s}=C_{s} \Delta\left[1-e^{-y^{+} / A^{+}}\right],
$$

where $A^{+}$is a constant usually taken to be $26 .^{38}$

It can also be seen in Eqs. (6) and (7) that the Smagorinsky model is purely dissipative and can account only for the energy being transferred from the resolved scales to the modeled scales. The transfer of momentum from the modeled scales to larger scales, known as back-scatter, has been shown to be present in a range of transitional and turbulent flows, ${ }^{40}$ and it has further been shown that failure to account for this effect can prevent the growth of perturbations in the velocity field that leads to boundary layer transition. ${ }^{41}$ The consequences of this are explored further in Sec. V.

The second subfilter scale model considered is the localized dynamic $\mathrm{k}$ model developed by Kim and Menon. ${ }^{42,43}$ This is a oneequation transport model for the subfilter scale turbulent kinetic energy. The idea of using a dynamic procedure to determine model coefficients has been applied to a number of models, for example, by Germano et al. ${ }^{44}$ for the determination of $C_{s}$ in the Smagorinsky model. The motivation behind this lies in the fact that the different turbulent scales can behave very differently. It stands to reason that the modeled scales will be most similar to the smallest resolved scales and so it is those that are used in determining the model constants for the SFS model. This is achieved by applying a test filter $\bar{\Delta}$ to the flow field, which is larger than the grid filter. This allows for only the smallest resolved scales to be used for the determination of the subfilter scale effects.

Following the approach set out by Kim and Menon, ${ }^{43}$ we begin by defining the SFS turbulent kinetic energy as

$$
k_{S F S}=\frac{1}{2}\left(\overline{u_{k} u_{k}}-\bar{u}_{k} \overline{u_{k}}\right) \text {. }
$$

The transport equation for this is given by

$$
\frac{\partial k_{S F S}}{\partial t}+\bar{u}_{i} \frac{\partial k_{S F S}}{\partial x_{i}}=-\tau_{i j} \frac{\partial \bar{u}_{i}}{\partial x_{j}}-\varepsilon_{S F S}+\frac{\partial}{\partial x_{i}}\left(v_{S F S} \frac{\partial k_{S F S}}{\partial x_{i}}\right) .
$$

The SFS dissipation rate, $\varepsilon_{S F S}$, is modeled using

$$
\varepsilon_{S F S}=C_{\varepsilon} \frac{k_{S F S}^{3 / 2}}{\bar{\Delta}} .
$$

The subgrid scale stress tensor is then given by

$$
\tau_{i j}=-2 C_{\tau} \bar{\Delta} k_{S F S}^{1 / 2} \bar{S}_{i j}+\frac{2}{3} \delta_{i j} k_{S F S} .
$$

The coefficients $C_{\tau}$ and $C_{\varepsilon}$ are determined using a dynamic procedure. The test filter is usually taken to be twice the grid filter ${ }^{43}$ and is denoted by $\widehat{\Delta}$. Using this, we derive the test-scale Leonard's stress,

$$
L_{i j}=\widehat{\overline{u_{i} u_{j}}}-\hat{\hat{u}_{i}} \hat{\hat{u}_{j}} \text {. }
$$

The resolved turbulent kinetic energy at the test filter level is therefore

$$
k_{\text {test }}=\frac{1}{2}\left(\widehat{\overline{u_{k} u_{k}}}-\hat{\overline{u_{k}}} \hat{\overline{u_{k}}}\right) .
$$

From this, the model constants are defined as

$$
C_{\tau}=\frac{L_{i j} \sigma_{i j}}{\sigma_{l m} \sigma_{l m}}
$$

where

$$
\sigma_{i j}=-\Delta k_{t e s t}^{1 / 2} \widehat{\overline{S_{i j}}}
$$

and

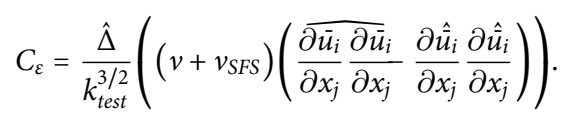

The model therefore contains no free parameters, with all coefficients being determined from the smallest resolved scales.

It only remains to define the filter width. This is most commonly related to the cell volume through

$$
\Delta=\sqrt[3]{h_{x} h_{y} h_{z}},
$$

where $h_{i}$ denotes the cell length in the $i_{\text {th }}$ direction. This approach introduces a problem for boundary layer flows, where high aspectratio cells are used in the near wall region. The use of Eq. (18) then leads to the filter width being less than the cell length in the streamwise direction, which introduces errors into the flow field. To avoid this problem, a different filter definition is used in this study,

$$
\Delta=\lambda \cdot \max \left(h_{x}, h_{y}, h_{z}\right), \lambda \in \mathbb{N} .
$$

The appropriate value for the filter-grid ratio, $\lambda$, depends on both the mesh resolution and the discretization scheme. Several studies have considered suitable values for the filter-grid ratio. ${ }^{29,45}$ These studies concluded that the filter width should be at least 4 times the cell width when a second-order scheme is used and 2 times the width when a sixth-order scheme is used. A value of $\lambda=4$ is used in this study unless otherwise stated.

\section{Numerical methods}

The general purpose finite-volume code OpenFOAM is used for all of the simulations in this study. The coupled pressure/velocity fields are solved at each time step using the PISO (pressure implicit with splitting of operators) algorithm with an algebraic multigrid solver for the pressure correction equation. The time derivative is discretized using a 3-point backward scheme.

For the direct numerical simulations, a second-order central scheme is used for the convective term, whereas several different methods are used for the LES. The central scheme is favorable because it exhibits lower dissipation than an upwind-biased scheme. However, it is prone to dispersion errors, particularly on coarser meshes. Because of this, it can be favorable to introduce a level of upwinding to stabilize the solution field. This can be achieved through a blended approach, which is considered below. For the convective flux through a face, denoted here as $\phi$, the general form of a second-order scheme is (see Fig. 1),

$$
\phi_{e}=\phi_{P}+\frac{1}{2} \psi(r)\left(\phi_{E}-\phi_{P}\right),
$$

where

$$
r=\frac{\phi_{P}-\phi_{W}}{\phi_{E}-\phi_{P}} .
$$

The function, $\psi(r)$, can be used to determine the level of blending. We define a weighting factor, $\alpha: \alpha \in[0,1]$, where $\alpha=0$ results in a pure central scheme. Using this, we can rewrite Eq. (20) as

$$
\phi_{e}=\phi_{P}+\frac{1}{2}(1-\alpha+\alpha r)\left(\phi_{E}-\phi_{P}\right) .
$$




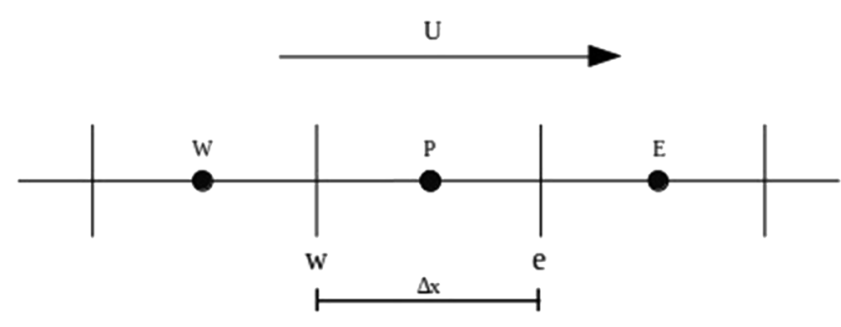

FIG. 1. Stencil showing nodes and faces for a 1D Cartesian grid.

TABLE II. Mesh parameters for 3D NACA0012 mesh. $g$ denotes the wall-normal growth ratio.

\begin{tabular}{lccccc}
\hline \hline Mesh & N cells $\left(\times 10^{-6}\right)$ & $\Delta x^{+}$ & $\Delta y^{+}$ & $\Delta z^{+}$ & $g$ \\
\hline A & 1.8 & 42 & 38 & 0.6 & 1.07 \\
B & 4.1 & 29 & 23 & 0.6 & 1.07 \\
C & 8.7 & 16 & 15 & 0.6 & 1.07 \\
D & 25 & 8 & 8 & 0.6 & 1.07 \\
E & 58 & 6 & 5 & 0.4 & 1.04 \\
\hline \hline
\end{tabular}

We consider three different values of $\alpha$ in this study: $\alpha=0,0.1,0.25$. Note that the latter results in the QUICK scheme.

\section{PROBLEM DEFINITION}

The numerical study is split into two parts. The first part considers fully resolved direct numerical simulations to allow for a detailed picture of the flow physics to be developed. Results from two highly refined meshes are compared, with excellent agreement seen. A number of large eddy simulations are presented in the second part, with the results being compared to the DNS. A total of 5 different mesh resolutions are considered, with grid-independence of the DNS data being demonstrated with the two highest resolution meshes (D and E in Table II). Large eddy simulations are carried out on meshes A, B, and C. The Smagorinsky and dynamic k SFS models are considered together with three different discretization schemes.

\section{A. Geometry and meshing}

The geometry under consideration is a NACA0012 airfoil with a chord length of $0.3 \mathrm{~m}$. The foil has a trailing edge bluntness equal
TABLE III. Comparison of mesh resolutions of similar studies.

\begin{tabular}{lcrccc}
\hline \hline References & LES/DNS & $\operatorname{Re}$ & $\Delta x^{+}$ & $\Delta y^{+}$ & $\Delta z^{+}$ \\
\hline 21 & LES & $3.5 \times 10^{5}$ & 45 & 30 & 0.8 \\
24 & LES & $6 \times 10^{4}$ & 25 & 13 & $<1$ \\
4 & DNS & $1 \times 10^{5}$ & $<13$ & $<15$ & $<1$ \\
3 & DNS & $5 \times 10^{4}$ & 3.4 & 6.5 & 1.0 \\
\hline \hline
\end{tabular}

to $0.25 \%$ of the chord. The trailing edge thickness is intended to represent a sharp but physically realistic form. The choice of span is a compromise between computational effort and the ability to fully resolve the three-dimensional boundary layer. As a guide, in Ref. 47, a span of $s=0.2 c$ is found to be adequate to capture the largest scales for the DNS of the flow over a foil in full stall. Other studies use a smaller span, for example, $s=0.16 c$ in Ref. 21 and $s=0.1 c$ in Ref. 4 . A span of $s=0.2 c$ is used in this study.

The domain extends 4 chord lengths upstream and 10 downstream. In the $\mathrm{z}$-direction, the domain extends 4 chord lengths above and below the foil. Preliminary studies found this to be adequate to prevent reflections off the boundaries.

The mesh designs are block-structured with a C-grid around the foil. Multiblock grading is used to allow for a better control over the refinement in key regions (see Fig. 2). 5 different mesh resolutions are considered, ranging from $1.8 \times 10^{6}$ to $58 \times 10^{6}$ elements. The parameters of each, given in wall units, are shown in Table II. The nondimensional mesh parameters have been estimated using the wall shear-stress from the Blasius boundary layer equation. The validity of this is assessed during the postprocessing. For comparison, the mesh parameters used in a number of other similar studies are given in Table III.

\section{B. Boundary and initial conditions}

A fixed value boundary condition is applied to the velocity field at the inlet, and a zero-gradient condition is applied at the outlet. The velocity magnitude is $\bar{U}=5 \mathrm{~ms}^{-1}$. A no-slip condition is imposed on the foil. For the pressure field, a zero-gradient condition is imposed at the inlet with a fixed-value condition at the outlet. For the LES, the turbulent quantities are set to zero on the foil and the inlet, with a zero-gradient condition imposed at the outlet. Note that this means that the freestream subfilter scale turbulent kinetic energy is zero.
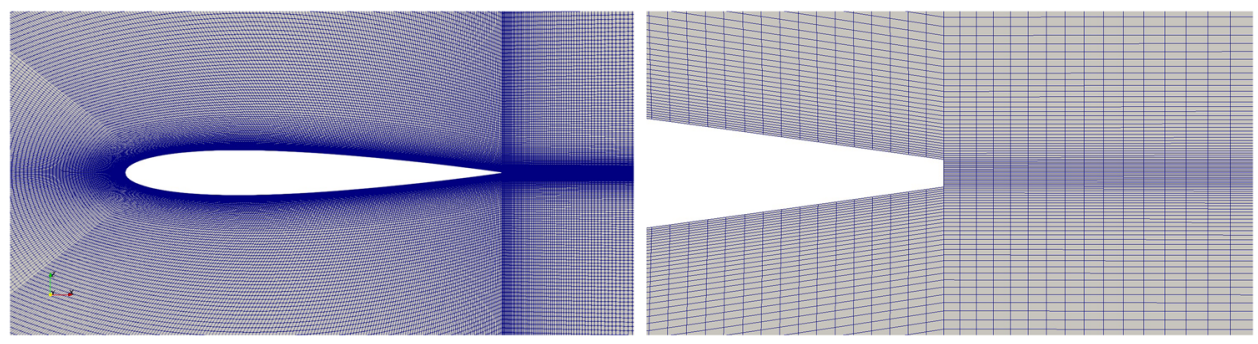

FIG. 2. Design of mesh $\mathrm{C}$ showing the mesh around the foil and at the trailing edge. 


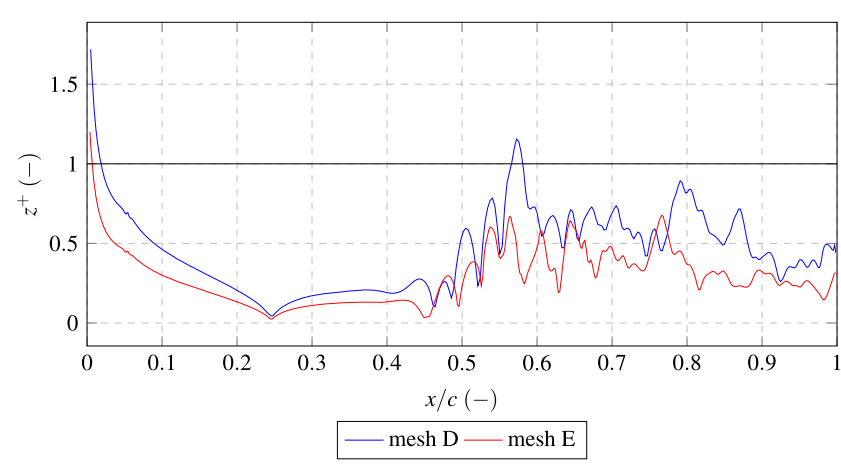

FIG. 3. Instantaneous $z^{+}$as a function of chord position on the suction side for time $t=1.8$.

The flow-field is initialized with a steady RANS computation using the $k-\omega$ SST turbulence model. This enables the computations to start with a more realistic flow-field which improves the stability of the calculation and also reduces the length of time needed to obtain statistically meaningful results.

The simulations are run for a total of $30 c / U$. The initial transients were found to have attenuated leaving a statistically stationary flow after approximately $12 c / U$. A fixed time step is chosen to satisfy the CFL condition. This required time steps of $3.33 \times 10^{-4} \mathrm{c} / U$ and $2.5 \times 10^{-4} \mathrm{c} / U$ for meshes $\mathrm{D}$ and $\mathrm{E}$, respectively. These simulations required approximately 50000 core-hours (on 256 cores) for mesh $\mathrm{D}$ and 230000 for mesh $\mathrm{E}$ (on 512 cores).

The validity of the mesh dimensions given in Table II has been assessed using the computed $z^{+}$(wall-normal) values over the foil. The mean $z^{+}$is 0.52 for mesh $\mathrm{D}$ and 0.31 for mesh $\mathrm{E}$, which is slightly lower than the estimated values. However, for mesh $\mathrm{D}$, the instantaneous $z^{+}$exceeds 1 at certain times due to velocity fluctuations where the boundary layer transitions but only by a small margin. For mesh

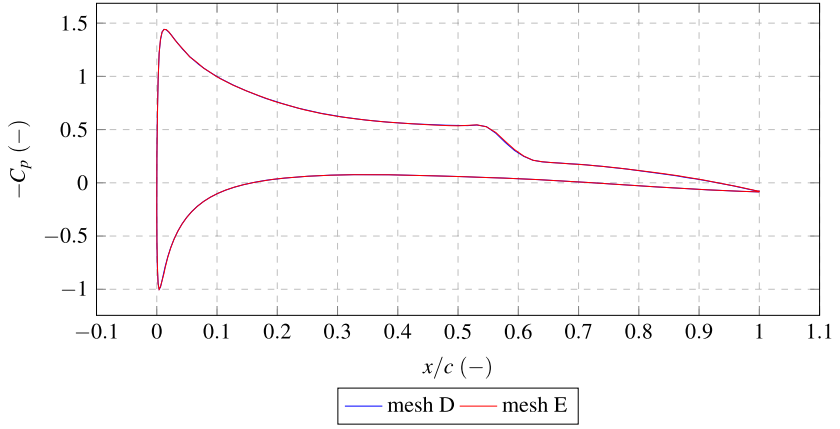

FIG. 5. Mean chordwise pressure coefficient at mid-span.

E, $z^{+}$remains below 1 apart from right at the leading edge. This is shown in Fig. 3. Both meshes exceed the criterion right at the leading edge on the suction side, but this is not thought to affect the results.

\section{DIRECT NUMERICAL SIMULATIONS}

\section{A. Mean flow}

The mean boundary layer flow is characterized by a thin separation bubble on the suction side. A small region of reversed flow exists close to the wall within this bubble until the reattachment point. This is illustrated in Fig. 4. The pressure side of the boundary layer remains attached and laminar along the entire chord length.

Excellent agreement is seen between the results from meshes D and $\mathrm{E}$ as is demonstrated for the mean pressure coefficient and mean boundary layer, as shown in Figs. 5 and 6.

The boundary layer profiles in Fig. 6 show that the first half of the boundary layer is characterized by a growing separation region. This then reattaches at around $x / c=0.56$, which

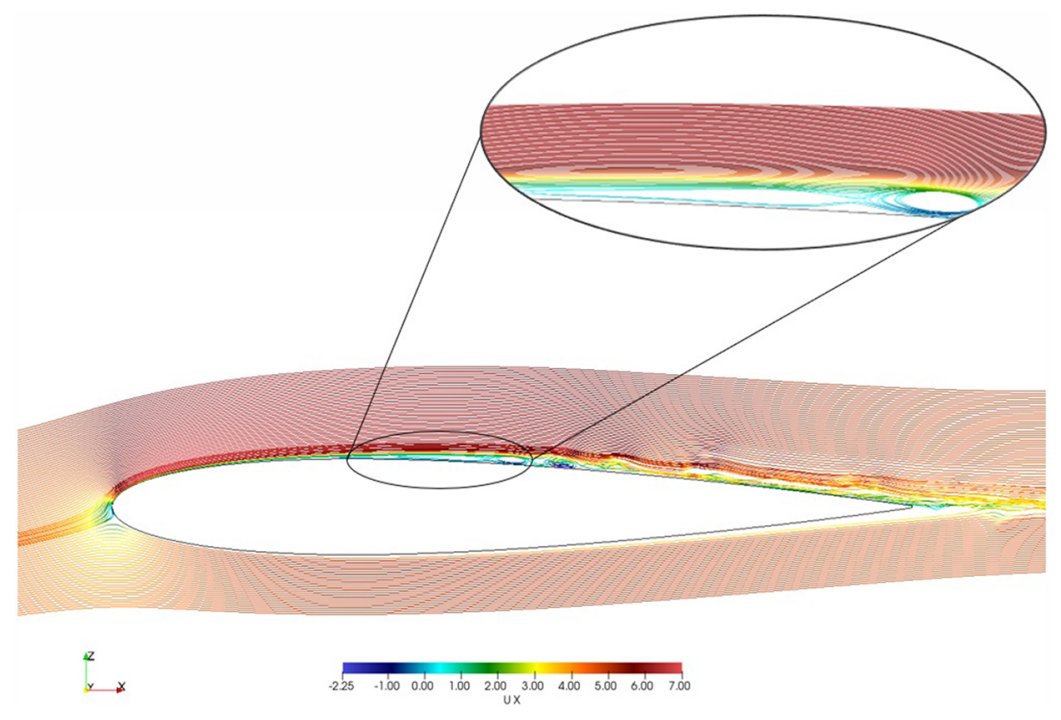

FIG. 4. Instantaneous flow lines illustrating the separation bubble and region of reversed flow on the suction side. 


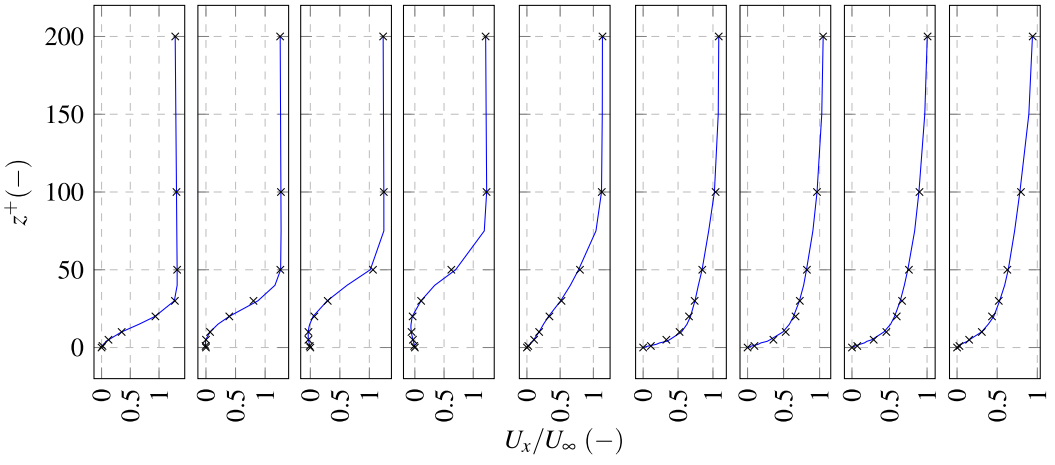

FIG. 6. Mean streamwise velocity $U_{x} / U_{\infty}$ in the boundary layer at $x / c=0.2,0.3, \ldots, 1.0$. The solid line represents mesh $\mathrm{E}$, and the markers $(\mathrm{x})$ represent mesh $\mathrm{D}$.

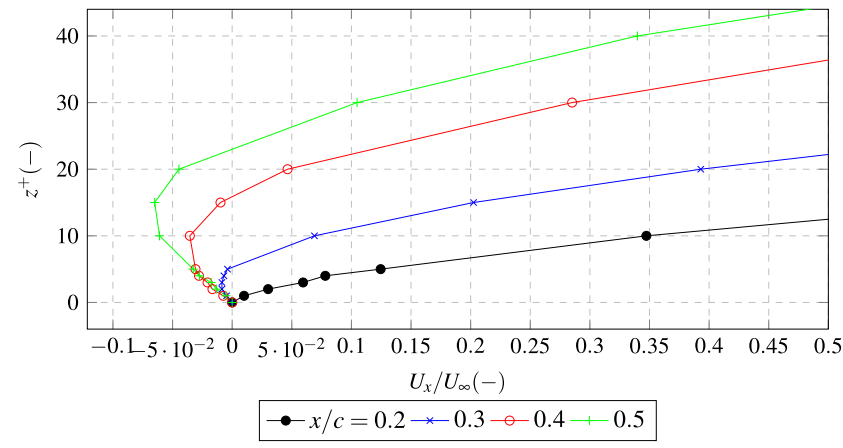

FIG. 7. Streamwise velocity showing the growth in the separation bubble over the first half of the airfoil. Results are from mesh $\mathrm{E}$. coincides with the later stages of the transition of the boundary layer. Beyond this, we see that the velocity profiles tend more toward the log-law profile associated with a fully turbulent boundary layer. The growth of the separation bubble can be seen more clearly in Fig. 7, which also shows the region of reversed flow close to the wall.

\section{B. Boundary layer transition}

The transition of the boundary layer occurs in several stages. The region of reversed flow close to the wall inside the separation bubble results in an unstable shear layer giving rise to a streamwise instability. Analysis of the velocity data in this region reveals that the amplitude of the fluctuations grows rapidly toward the end of the bubble, as shown in Figs. 8 and 9.
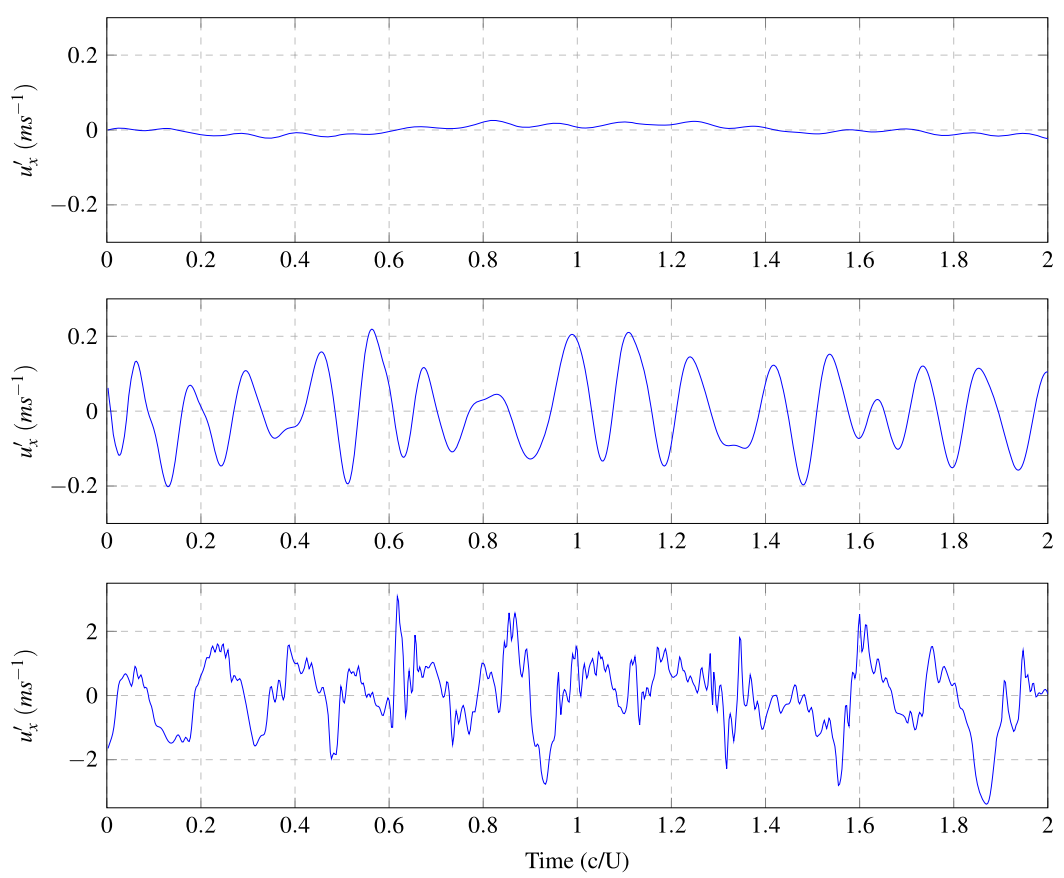

FIG. 8. Streamwise velocity fluctuations in the boundary layer for $x / c=0.4,0.5,0.6$ over two through-flow times. Note that the third plot has an expanded range on the $y$-axis. 

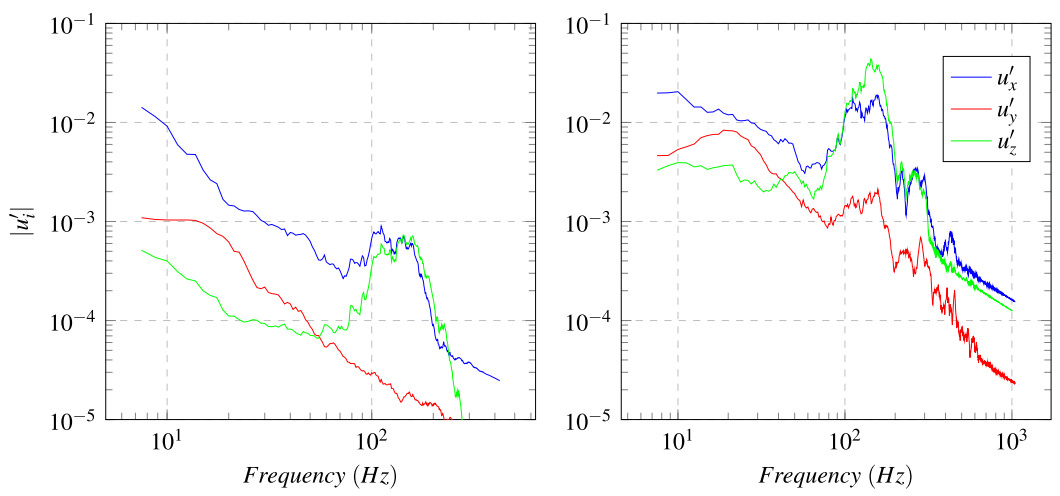

FIG. 9. Spectral plots of the streamwise and spanwise velocity fluctuations at $y^{+}=30$ for $x / c=0.4,0.5$.

Spectral analysis reveals further insights into the growth of the fluctuations during the early stages of transition. Figure 9 shows that the initial disturbance is dominated by a narrowband component centered around $140 \mathrm{~Hz}$, which grows rapidly between $x / c=0.4$ and $x / c=0.5$. During the initial transition, the amplitude of the streamwise and wall-normal fluctuations is far greater than for the spanwise fluctuations. However, it can be seen that when the spanwise fluctuations start to develop toward the end of the separation bubble, their structure is similar to that of the streamwise fluctuations, indicating a degree of coupling, possibly through $u_{z}^{\prime}$. Between $x / c=0.4$, where the fluctuations are first seen, and $x / c=0.6$, the normalized rms of the streamwise fluctuations (normalized by freestream velocity) grows from 0.0024 to 0.2106 , which is an increase of almost 2 orders of magnitude.

It is often the case that the early stages of boundary layer transition are due to some external parameter, such as low-intensity free-stream turbulence, surface roughness, or an imperfection in the geometry. However, the shear layer in the near-wall region combined with the recirculation of the flow inside the bubble appears to provide this initial perturbation, meaning that no external action is needed for the transition to occur. This process follows that of a Kelvin-Helmholtz instability.

After the boundary layer reattaches at approximately $x / c$ $=0.56$, the structure of the fluctuations becomes increasingly chaotic and the narrowband component disappears. Once the boundary layer becomes fully turbulent, the spanwise fluctuations increase sharply but remain lower than the streamwise fluctuations at lower wavenumbers. At higher wavenumbers, the amplitudes in each direction are much closer, indicating an increasing level of isotropy for the smaller scales, whereas the larger, energy containing scales are more anisotropic. This can be seen in Fig. 10.

The fluctuations of the surface pressure also show a sharp growth in narrowband fluctuations followed by an increasingly broadband spectrum. The surface pressure spectra are shown in Fig. 11 for both meshes $\mathrm{D}$ and $\mathrm{E}$, again showing excellent agreement.

Analysis of the boundary layer also reveals that the turbulent kinetic energy grows to a maximum around the point where the boundary layer becomes fully turbulent and then decays toward the trailing edge. This also shows a very sharp growth between $x / c=0.5$ and $x / c=0.6$. Results for both meshes $\mathrm{D}$ and $\mathrm{E}$ are shown in Fig. 12, again indicating excellent agreement between the 2 meshes. Figure 12 also shows that the highest turbulent kinetic energy is found in the boundary layer region $30<y^{+}<50$.

An overview of the transition process can also be seen by looking at the vorticity over the foil. Figure 13 shows this using a contour plot of the $Q$-criterion, ${ }^{48} Q=\frac{1}{2}\left(|\Omega|^{2}-|S|^{2}\right)$, where $\Omega$ and $S$ denote the vorticity and rate-of-strain tensors, respectively. A 2-dimensional tube vortex structure can be seen where the transition process begins which quickly breaks down into 3-dimensional vortical structures which are convected over the trailing edge.

We can summarize the following transition process:

1. Flow separation close to the leading edge leads to the formation of a laminar separation bubble on the suction side.
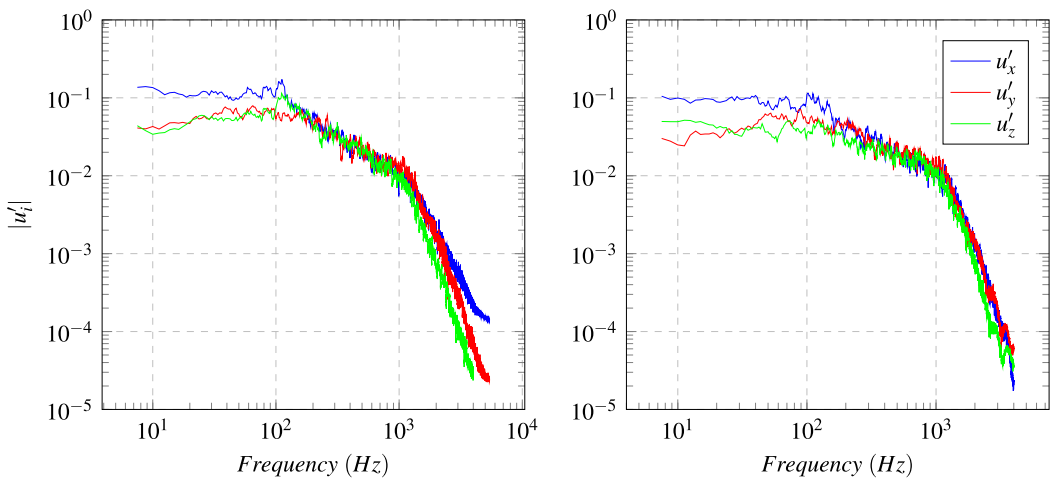

FIG. 10. Spectral plots of the streamwise and spanwise velocity fluctuations at $y^{+}=30$ for $x / c=0.6,0.7$. 

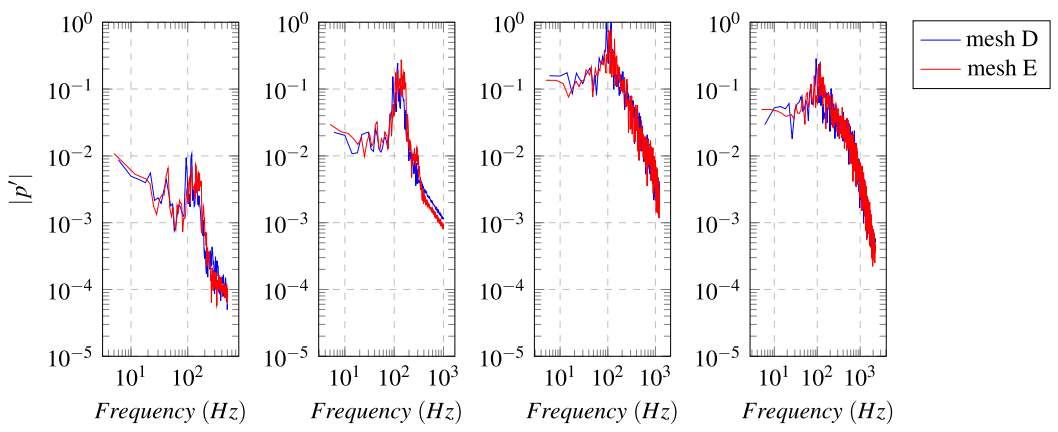

FIG. 11. Spectral plots of the mid-span surface pressure

fluctuations for $x / c=0.4,0.5,0.6,0.7$.

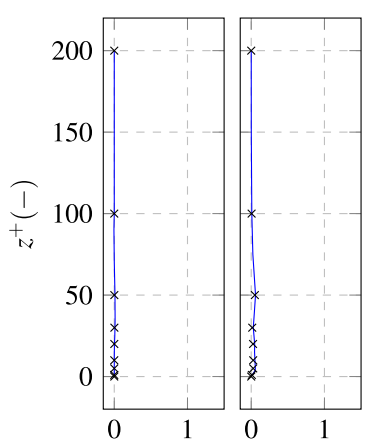

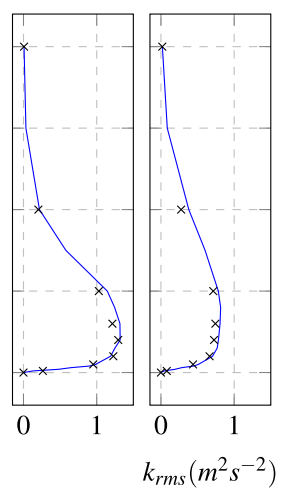
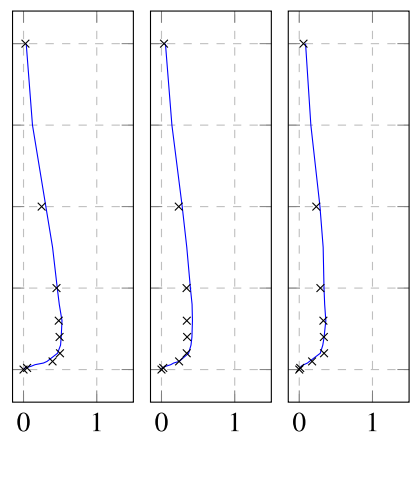

FIG. 12. RMS turbulent kinetic energy in the boundary layer at $x / c=0.4,0.5, \ldots, 1.0$. The solid line represents mesh $E$, and the markers $(x)$ represent mesh $D$.
2. A streamwise instability grows in the bubble very close to the wall, dominated by a narrowband component at $140 \mathrm{~Hz}$.

3. Small spanwise fluctuations start to appear shortly before the reattachment of the boundary layer.

4. The boundary layer reattaches coinciding with significant growth in fluctuations in all directions. The narrowband component in the streamwise fluctuations disappears.

5. The boundary layer becomes fully turbulent with no narrowband component and is convected over the trailing edge.

\section{LARGE EDDY SIMULATIONS}

To begin with, the performance of the two subfilter scale models is considered, followed by an assessment of the different discretization schemes.

\section{A. Effect of sub-filter scale model}

Significant differences are observed between the Smagorinsky model and the dynamic $\mathrm{k}$ model. Simulations conducted using the Smagorinsky model do not show any transition, and so the boundary layer remains laminar on both the pressure and suction sides of the foil. This is irrespective of the discretization scheme and mesh resolution. For all three meshes, there is a separated region close to the trailing edge on the suction side which expands as the mesh is refined, as shown in Fig. 14. Convergence toward the DNS data is not seen for the simulations using the Smagorinsky model for the three meshes used. All such simulations exhibit trailing edge flows consistent with laminar boundary layers, with tonal fluctuations corresponding to von Kármán vortex shedding. This behavior contrasts sharply with that seen for the dynamic $\mathrm{k}$ model (see Fig. 15), which does capture a transition in all cases.

To explain the differences in the flow fields resolved by the two models, we consider the subfilter scale viscosity in the boundary layer at $x / c=0.4,0.5,0.6$, which covers the region over which the transition should occur. This is shown in Fig. 16. The dynamic $\mathrm{k}$ model is effectively inactive in the boundary layer at $x / c=0.4$, with the subfilter scale viscosity more than 2 orders of magnitude lower than the molecular viscosity. The Smagorinsky model predicts a peak subfilter scale viscosity of $6 \times 10^{-5}$, which is 4 times larger than the molecular viscosity. This prevents the growth of any disturbance in the boundary layer and so prevents the transition from occurring. The dynamic $\mathrm{k}$ model only becomes active at $x / c=0.6$, which corresponds to the point at which the smaller turbulent scales

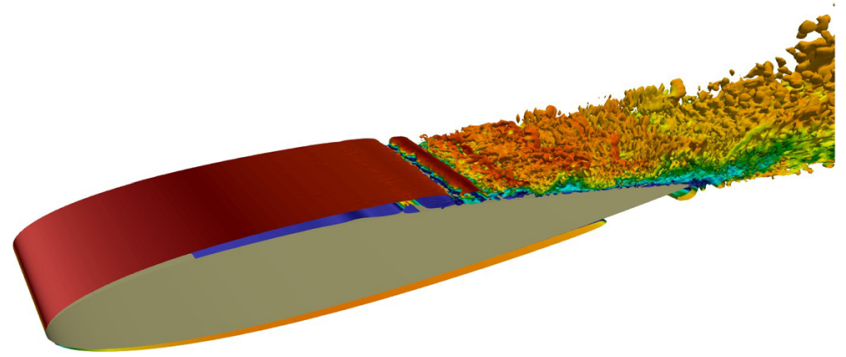

FIG. 13. Isocontours of $Q=100$ showing the transition of the boundary layer. Contours colored by velocity magnitude. 


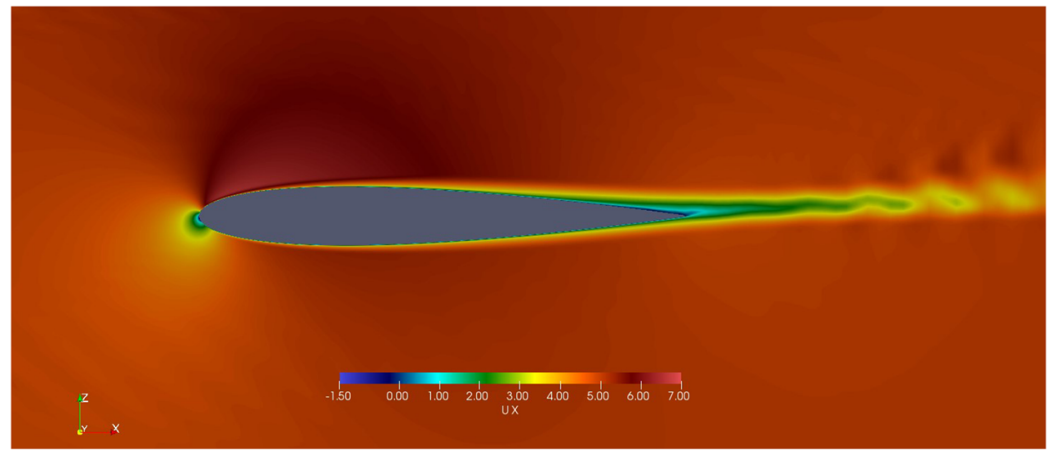

(a)

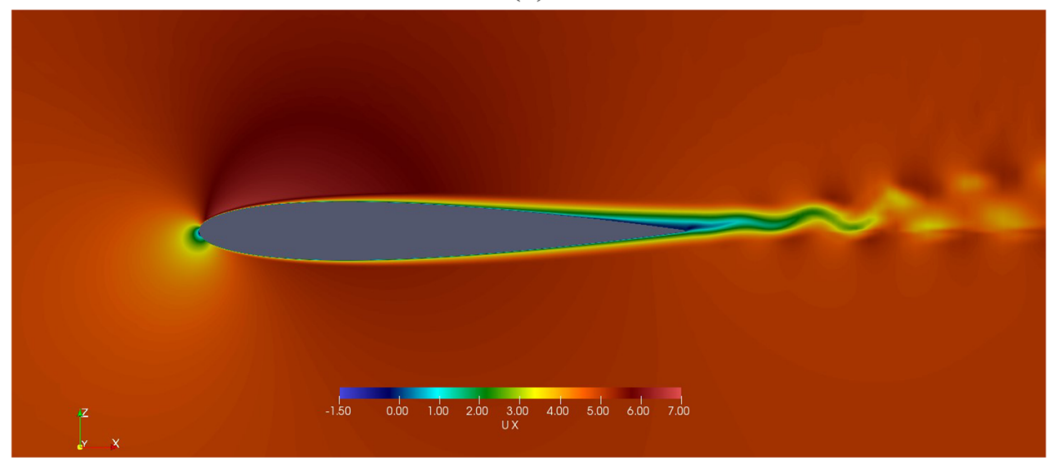

(b)

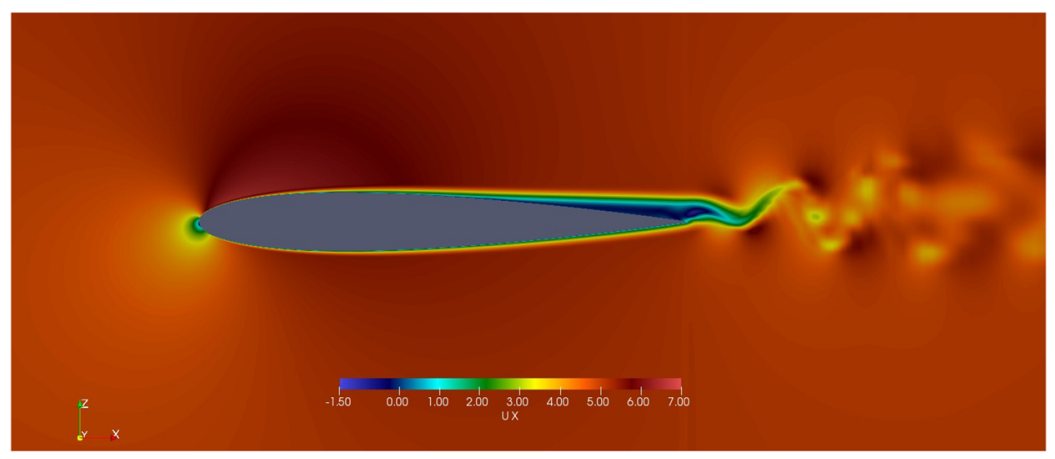

(c)
FIG. 14. Instantaneous streamwise velocity contours at midspan for (a) mesh A, (b) mesh B, and (c) mesh C with the Smagorinsky subfilter scale model. appear in the flow (as shown in Fig. 10). This suggests that this model is behaving correctly in that it is not damping out the larger scales in the boundary layer. The formulation of the Smagorinsky model is such that the subfilter scale viscosity must reduce with increasing mesh resolution and this is shown in Fig. 17. However, based on the rate of reduction in $v_{S F S}$, it can be concluded that, without modifying $C_{s}$, the mesh would have to be refined beyond that required for the DNS in order to capture the transition process.

The direct proportionality of the turbulent lengthscale to the filter width in the Smagorinsky model also leads to difficulties with the damping function applied at the wall. Increasing the filter-grid ratio results in the sub-filter-scale viscosity being too large in the near-wall region. This was found to lead to nonphysical boundary layer behavior for larger filter-grid ratios. A solution to this might be to increase
$A^{+}$to force $v_{S F S} \rightarrow 0$ at the wall, but such an approach would be, to some degree, arbitrary.

The Smagorinsky coefficient could of course be reduced, either in the near-wall region or for the entire domain. This would reduce the subfilter scale viscosity and may allow for the transition to be captured. The same is true for the near-wall treatment. However, the required change to any model coefficient depends on the stage of the transition. During the initial stages, we require that $v_{S F S} \ll v_{m o l}$. As turbulence develops, $v_{S F S}$ needs to increase in response to the growth of the subfilter scales. The ability to do this is a key benefit of using the dynamic $\mathrm{k}$ model, where the local resolved flow field is used to determine the model coefficients.

The failure to capture the transition has a number of consequences, both for the prediction of global forces such as lift and drag 


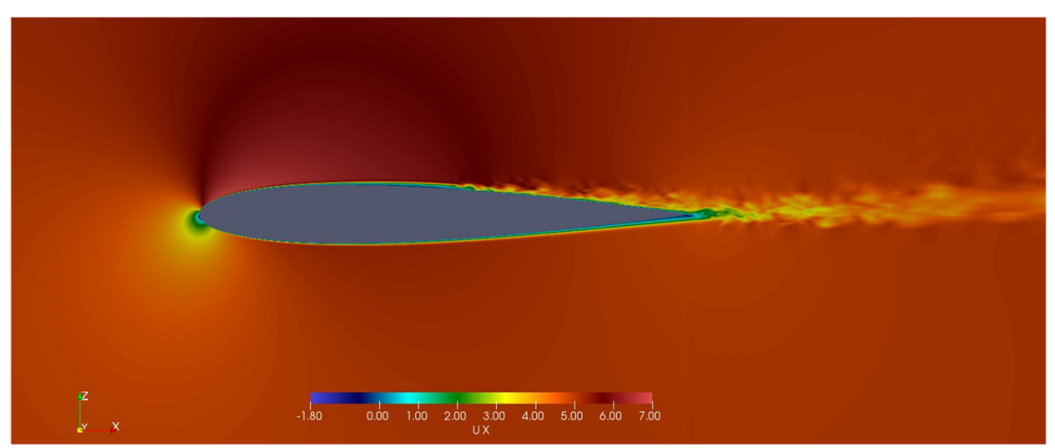

(a)

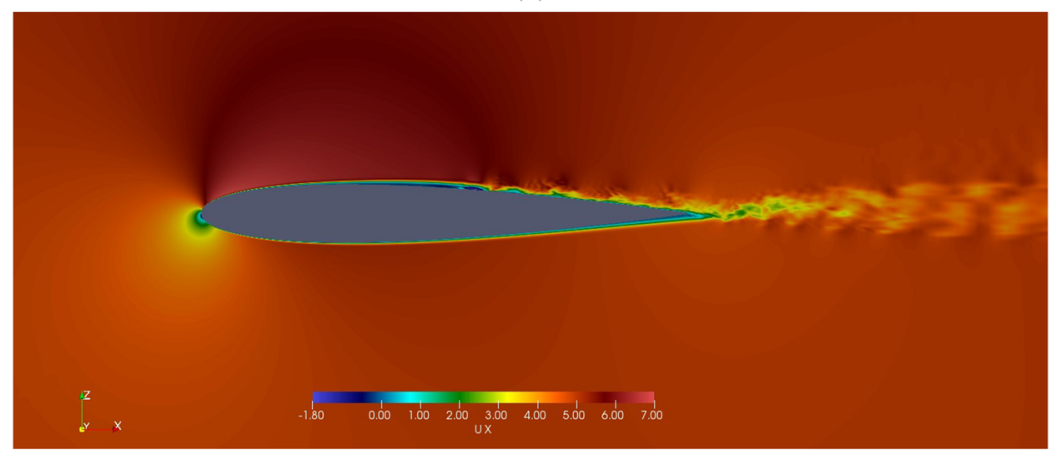

(b)
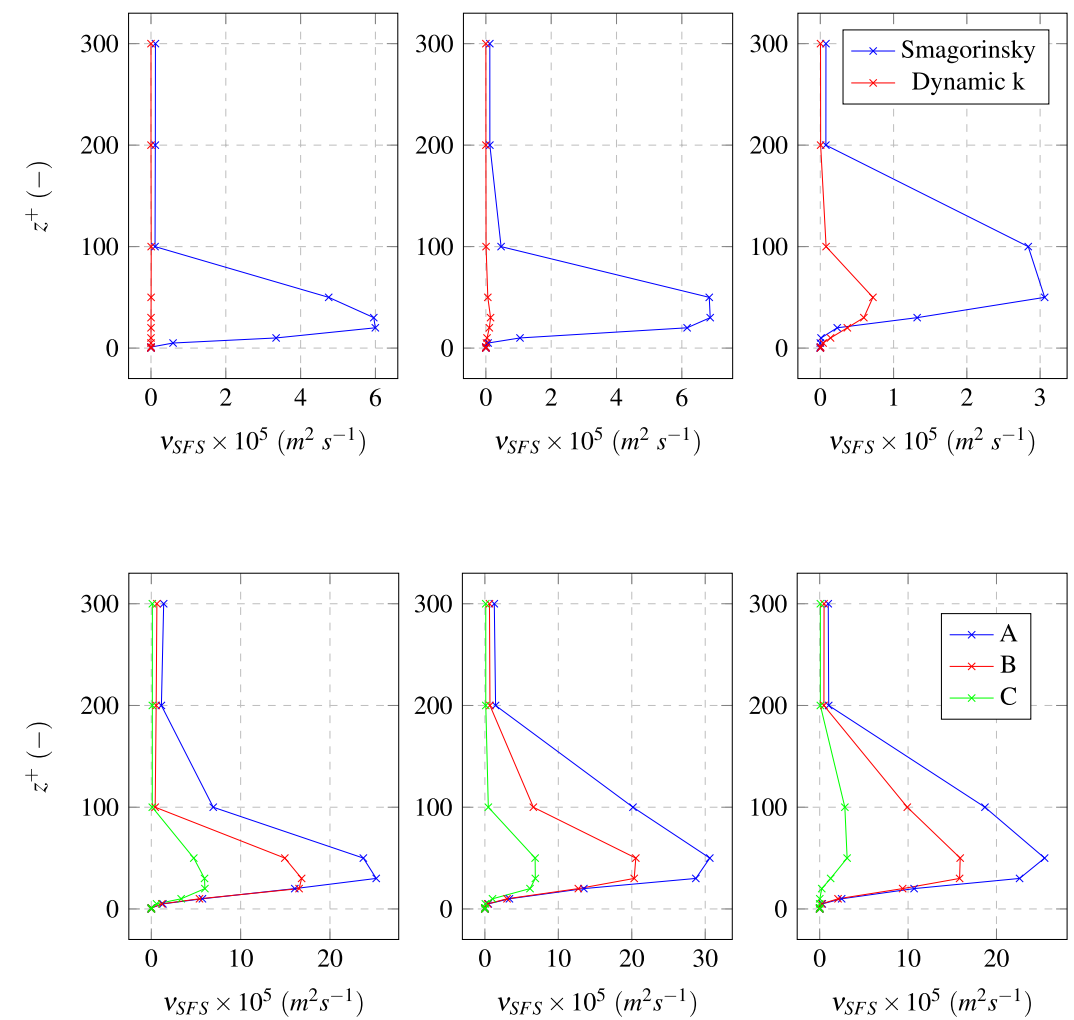

FIG. 15. Instantaneous streamwise velocity contours for (a) mesh C, LES with the dynamic k model and (b) mesh $D$, DNS.

FIG. 16. Comparison of the mean subfilter scale viscosity in the boundary layer at $x / c=0.4,0.5,0.6$ for the two SFS models. Results are for mesh $C$ using a pure central scheme.

FIG. 17. Mean SFS viscosity determined by the Smagorinsky model in the boundary layer at $x / c=0.4,0.5,0.6$. 

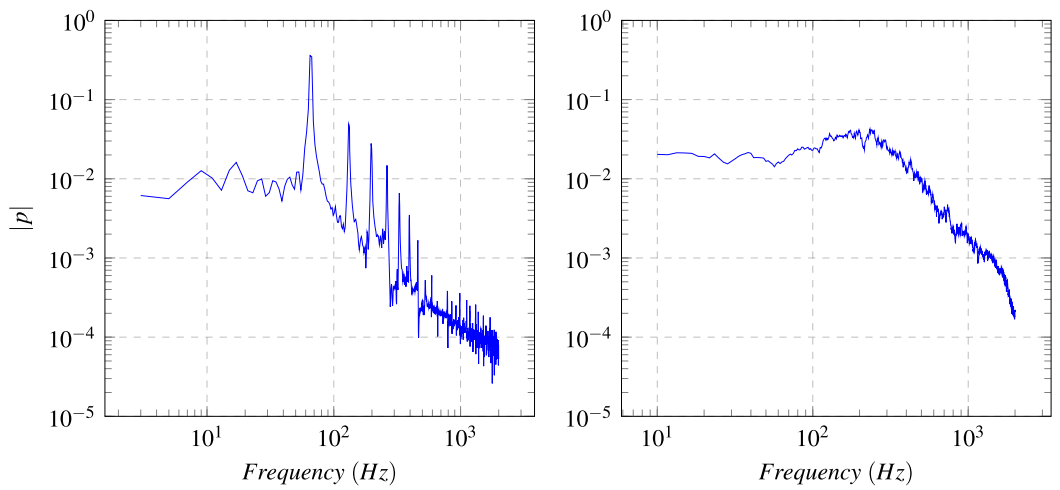

FIG. 18. Trailing edge pressure spectra for the Smagorinsky model (left) and the dynamic k model (right). Results are taken from simulations using mesh $\mathrm{C}$ with a pure central scheme.

and also on the prediction of the trailing edge flow. The separation region at the trailing edge predicted by the Smagorinsky model leads to a large overprediction in the drag and an under-prediction in the lift. Significant differences are also seen in the pressure spectra at the trailing edge, which is shown in Fig. 18. The pressure spectra at the trailing edge consist primarily of discrete frequencies at multiples of $65 \mathrm{~Hz}$ when the Smagorinsky model is used, whereas no discrete frequencies are observed for simulations conducted using the dynamic $\mathrm{k}$ model, which is in agreement with the DNS data.

\section{B. Effect of mesh resolution and discretization scheme}

The previous analysis has shown that the Smagorinsky model, in its standard form, is unsuitable for resolving transitional flows and so is not considered further in this study. The local dynamic $\mathrm{k}$ model has shown that it does allow for the transition from laminar to turbulent to occur and so we must now consider how well simulations using this model capture each stage of the transition and the resulting turbulent boundary layer.

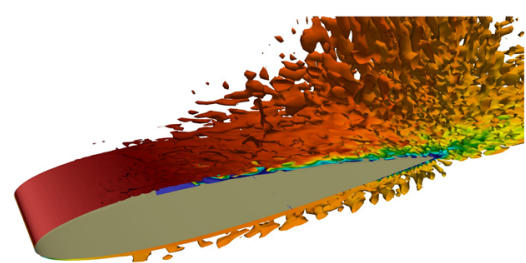

(a) Mesh A, CDS

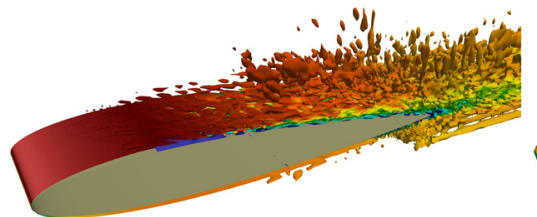

(c) Mesh B, CDS

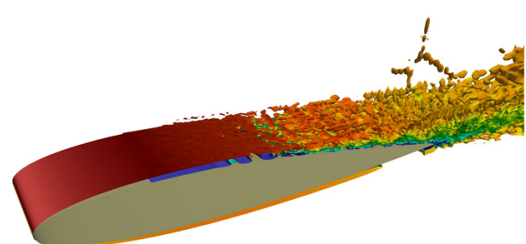

(e) Mesh C, CDS

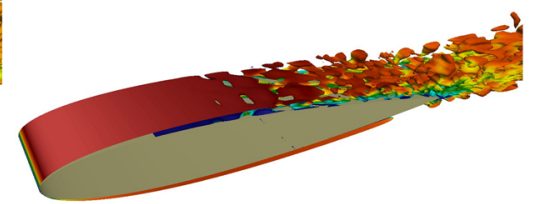

(b) Mesh A, 75-25 blended scheme

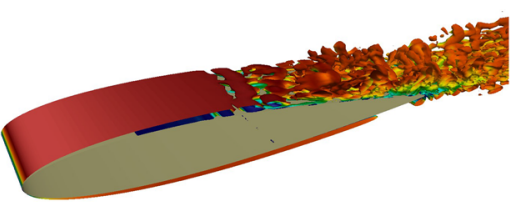

(d) Mesh B, 75-25 blended scheme

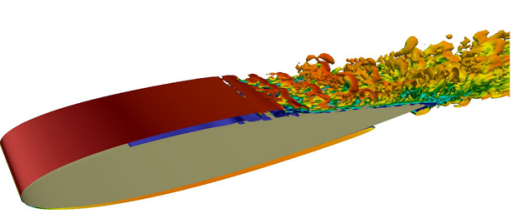

(f) Mesh C, 75-25 blended scheme

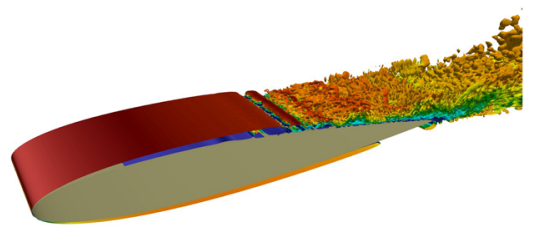

FIG. 19. Isocontours of $Q=100$ colored by velocity magnitude. (a)-(f) show results from large eddy simulations, and (g) represents direct numerical simulations.

(g) Mesh D, DNS 


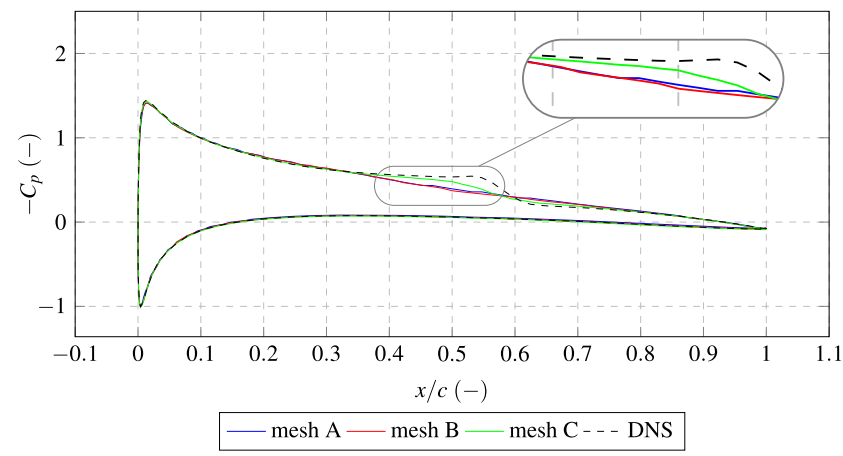

FIG. 20. Mean pressure coefficient over the airfoil for the dynamic $k$ model with pure central differencing. DNS results shown for comparison.

An overview of the qualitative differences in the mesh resolution and discretization schemes can be obtained by considering the isocontours of Q, shown in Fig. 19. For both of the discretization schemes shown, the flow field appears to converge toward the DNS data as the mesh is refined but there are marked differences in the results. The central difference scheme gives rise to significant dispersion errors for the coarser meshes, which manifest as unphysical fluctuations in the flow field. These appear predominantly toward the trailing edge but are also present in the region where the separation bubble should be. These errors are not visible in the vorticity plots for the 75-25 blended scheme, and a more expected convergence pattern is seen, with increasingly small turbulent structures being resolved as the mesh is refined.

The DNS data show the transition process beginning with a streamwise disturbance in the shear layer that results from the region of reversed flow close to the wall. Therefore, in order for the LES to capture the transition correctly, the bubble and region of reversed flow must be adequately resolved. Figures 20 and 21 show that the pure central scheme fails to capture the separation bubble for the 2 coarsest meshes, whereas the blended schemes show a separation bubble with a region of reversed flow for all three mesh resolutions. The predicted bubble size and the magnitude of the reversed flow increase as the mesh is refined, with the results converging toward the DNS data. For a given level of resolution, the 75-25

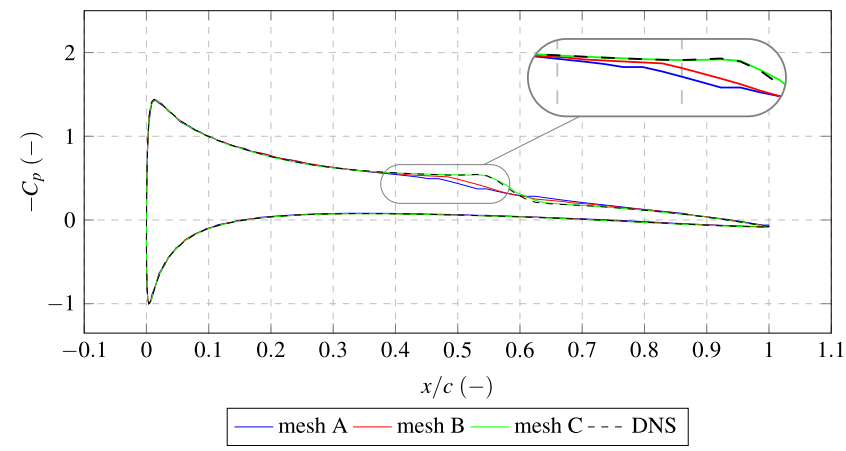

FIG. 21. Mean pressure coefficient over the airfoil for the dynamic $k$ model with 75-25 blending. DNS results shown for comparison.

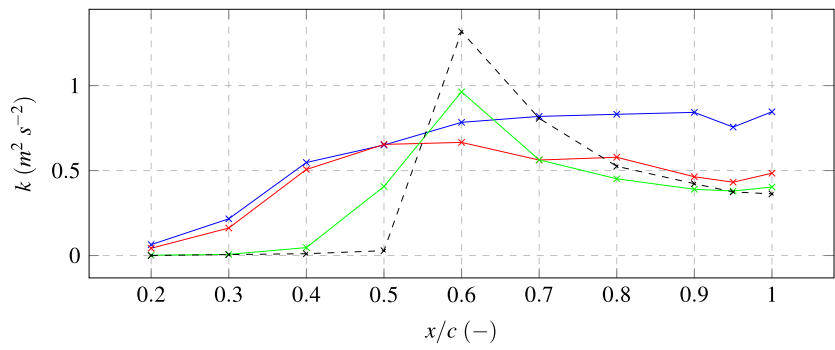

$* \operatorname{mesh} \mathrm{A} \approx-\operatorname{mesh} \mathrm{B} \approx-\operatorname{mesh} \mathrm{C}-*-\mathrm{DNS}$

FIG. 22. Resolved rms turbulent kinetic energy at $y^{+}=30$. DNS compared with the LES with pure central differencing.

blended scheme produces the most accurate representation of the bubble. Even when using mesh $\mathrm{C}$, the central scheme only allows for a very small region of reversed flow to be captured, whereas results from the blended scheme agree well with the DNS data at this resolution.

The failure of the coarser meshes when combined with a pure central scheme to capture the separation bubble can be explained by considering the vortical structures shown in Fig. 19. These indicate the presence of velocity fluctuations in the flow field that are upstream of where the transition should take place. The predicted levels of resolved turbulent kinetic energy in the boundary layer (see Figs. 22 and 23) show that the transition process predicted by the large eddy simulations is more gradual and begins further upstream than is predicted by the DNS. This is particularly true of the coarse meshes with pure central differencing.

This result should be considered in the context of the fundamental basis of large eddy simulation, which would suggest that coarse meshes might fail to resolve the small-scale instabilities that lead to the transition and either delay or prevent the transition from occurring. Clearly this behavior is not seen and the results generally show the coarser meshes predicting an earlier transition. This transition also occurs irrespective of whether the separation bubble forms, and so we must consider what other mechanisms are responsible for the behavior.

A more detailed understanding of this can be obtained by considering how the fluctuations evolve over the chord length. Figure 24

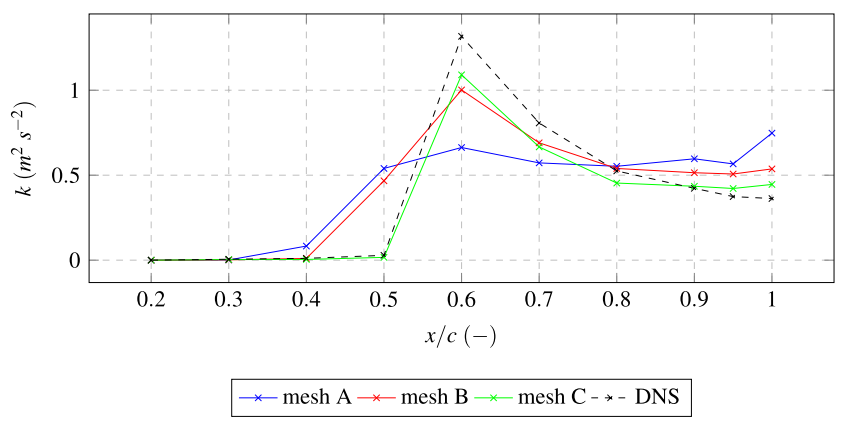

FIG. 23. Resolved rms turbulent kinetic energy at $y^{+}=30$. DNS compared with the LES with the $75-25$ blended scheme. 

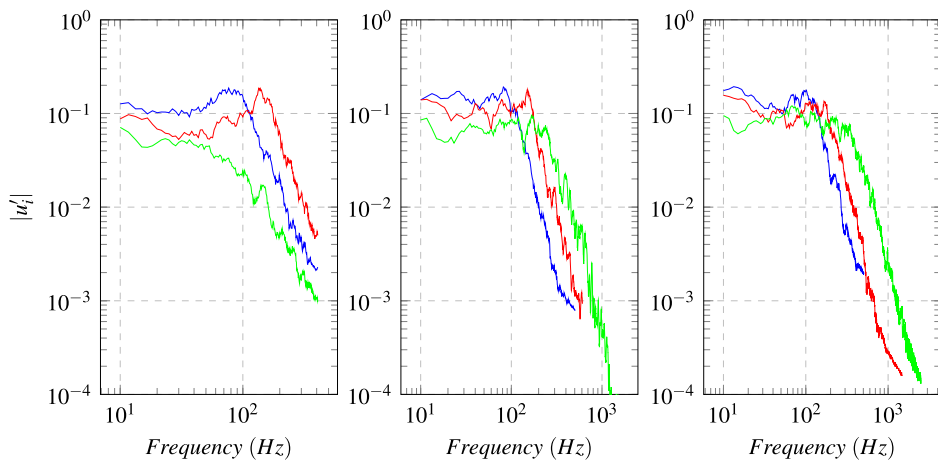

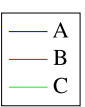

FIG. 24. Spectral plots of the streamwise velocity fluctuations at $y^{+}=30$ for $x / c=0.4,0.5,0.6$ for mesh $\mathrm{A}$, central scheme. shows the spectra of the streamwise velocity fluctuations for simulations using a pure central scheme. The behavior is clearly very different from that seen in the DNS. The boundary layer already contains significant fluctuations at $x / c=0.4$ with very little periodicity. A growth in the fluctuations is observed for mesh $\mathrm{C}$, but no narrowband component is present. The fluctuations are an order of magnitude higher at $x / c=0.4$ than predicted by the DNS.

The earlier transition seen in these simulations can be attributed to the dispersion errors in the flow field. Where the laminar separation bubble does not form, it is because that the boundary layer is already turbulent, with the transition having occurred upstream. As we have seen, the dispersion errors manifest as fluctuations in the flow field and so behave, in some sense, like freestream turbulence. This, in turn, causes the early transition to occur through a mechanism more like bypass transition.

The introduction of a small blending factor reduces the dispersion errors in the flow field and allows for the correct transition mechanism to occur. As the mesh is refined from $\mathrm{A}$ to $\mathrm{C}$, and as the blending factor is increased, the fluctuations at $x / c=0.4$ reduce. With a $10 \%$ level of up-winding (see Fig. 25), no fluctuations are seen at $x / c=0.4$ for mesh C. Increasing this to a $25 \%$ level of upwinding removes fluctuations from mesh B (Fig. 26). Therefore, the artificial dissipation introduced by the up-winding helps to remove the dispersion errors in the pretransitional and transitional region.

Convergence toward the DNS data can therefore be broken down into stages. First, as the mesh is refined or the blending is
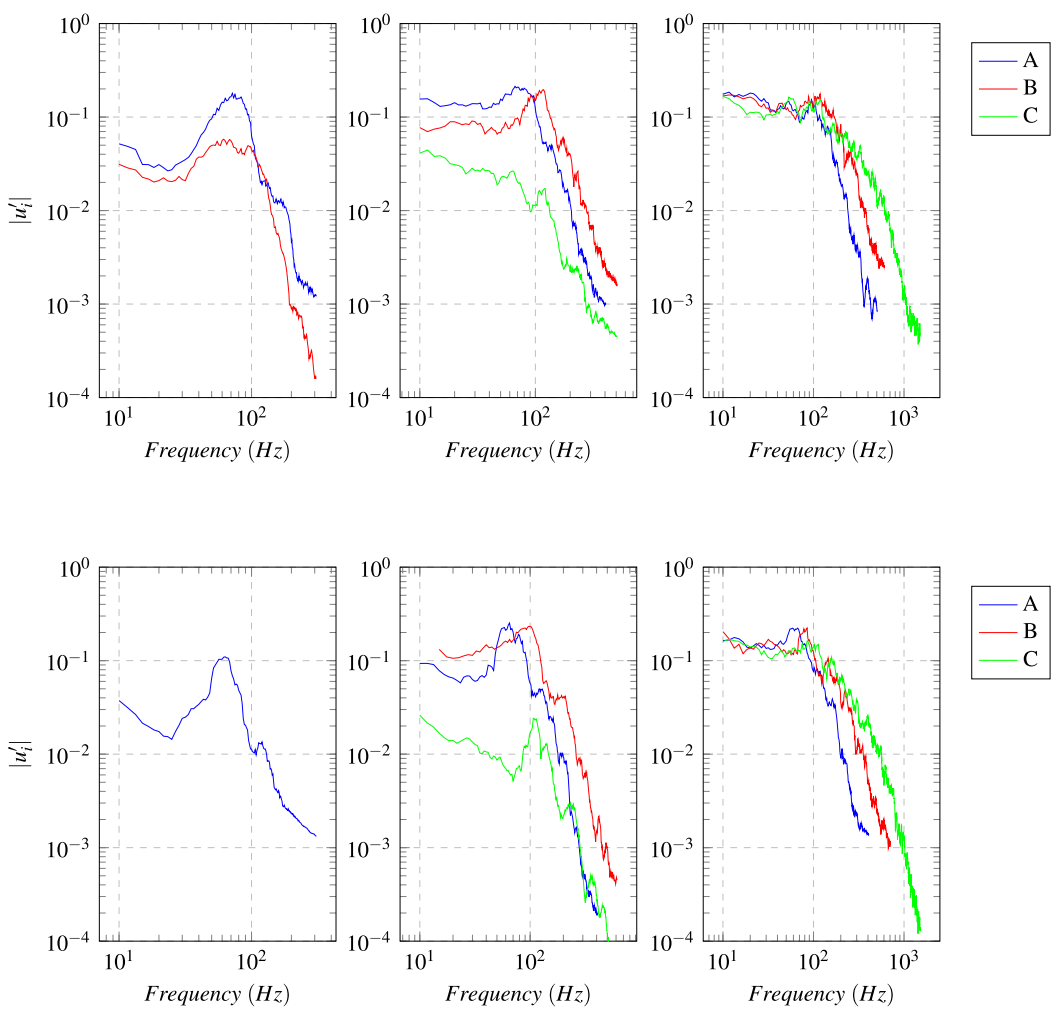

FIG. 25. Spectral plots of the streamwise velocity fluctuations at $y^{+}=30$ for $x / c=0.4,0.5,0.6$ for meshes $A, B$, and $C$ using the $90-10$ blended scheme.
FIG. 26. Spectral plots of the streamwise velocity fluctuations at $y^{+}=30$ for $x / c=0.4,0.5,0.6$ for mesh $C, 75-25$ blended scheme. 


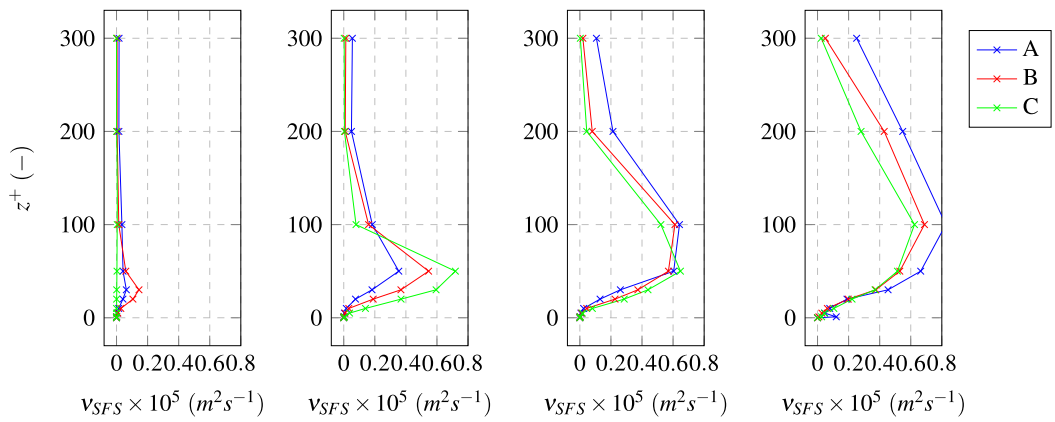

FIG. 27. Comparison of the mean subfilter scale viscosity in the boundary layer at $x / c=0.4,0.6,0.8,1.0$. Results are for meshes $A, B$, and $C$ using a central scheme.

increased, the dispersion errors responsible for an early transition are reduced. If these errors are reduced enough, the transition will occur by the correct mechanism but the initial stage may not be well captured and transition will be delayed. Refining the mesh further still moves this point upstream toward the location predicted by the DNS.

It is interesting to note that by $x / c=0.6$, all of the simulations using blended schemes predict similar levels of fluctuations at low wavenumbers, with increasing levels of dissipation observed at higher wavenumbers as the mesh becomes coarser. This shows that once the boundary layer has become fully turbulent and providing that the dispersion errors are minimal, the simulations behave as expected, with increased mesh refinement providing increased resolution.

It is important to recognize that the artificial dissipation introduced by blending is also a type of error. While the effect is positive in the pretransitional and transitional stages, the negative effects can be seen once the boundary layer is fully turbulent. Here, the effect is to damp out the higher-wavenumber fluctuations as can be seen at $x / c=0.6$. However, the dissipative effects are observed mainly at higher frequencies than those seen during the early stages of the transition. This, combined with the earlier results, shows that the artificial dissipation introduced by the blending does not prevent or delay the transition from occurring. This highlights one of the benefits of using spectral analysis as part of any verification as it allows for the wavenumber at which artificial dissipation becomes noticeable to be determined.

The interactions between the discretization scheme and the subfilter scale model also merit closer attention. In the pretransition stage, a decrease in subfilter scale activity is seen with increased blending (see Figs. 27 and 28). This is consistent with the increased dissipation that comes with an increased level of up-winding. In this region, such behavior is favorable as the subfilter scale activity here should be minimal. The SFS activity that does occur at $x / c=0.4$ is brought about in response to the presence of erroneous velocity fluctuations, which in this case result from the discretization scheme. The spectral analysis shows that this results in fluctuations over a wide range of scales, whereas the dynamic k SFS model is effective only at higher wavenumbers. Therefore, the subfilter scale model does not dissipate these fluctuations.

At $x / c=0.6$, the boundary layer is in the final stages of transition, with three-dimensional turbulent structures dominating. Interestingly, for all discretization schemes, we see an increase in the subfilter scale viscosity as the mesh is refined. Only at the trailing edge is the expected pattern observed, with reduced subfilter scale activity for more resolved meshes. This behavior again highlights the complex interaction between the SFS model, the discretization schemes, and the mesh resolution. This also shows that the artificial dissipation introduced by the discretization schemes used here does not replace the role of the SFS model, as is the case for the implicit LES.

We finally wish to assess how well each simulation has predicted the trailing edge flow. In Figs. 22 and 23, it can be seen that the coarser meshes overpredict the resolved turbulent kinetic energy here. The preceding analysis of the boundary layer transition process shows that this is because of the earlier transition that occurs due to the dispersion errors in the flow.

Spectral analysis of the resolved turbulent kinetic energy at the trailing edge shows that the overprediction occurs at low wavenumbers. The spectral content of the resolved turbulent kinetic energy as
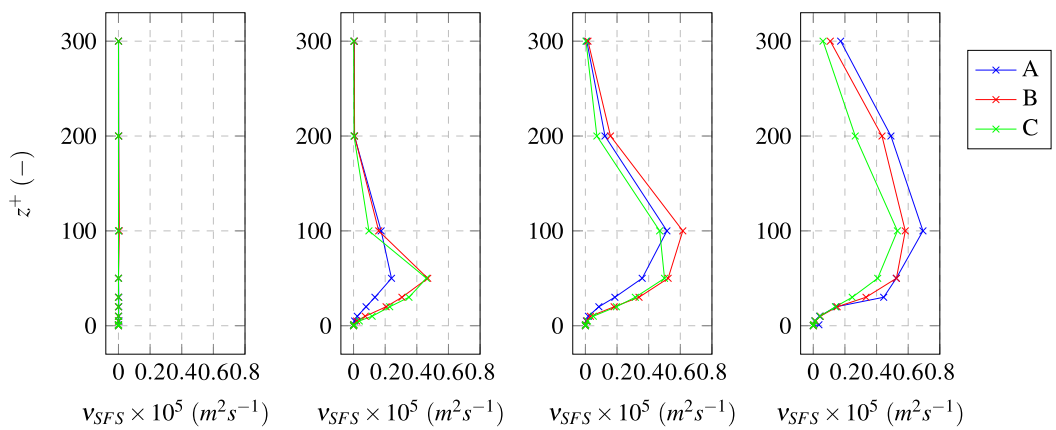

FIG. 28. Comparison of the mean subfilter scale viscosity in the boundary layer at $x / c=0.4,0.6,0.8,1.0$. Results are for meshes $\mathrm{A}, \mathrm{B}$, and $\mathrm{C}$ using a $75-25$ blended scheme. 

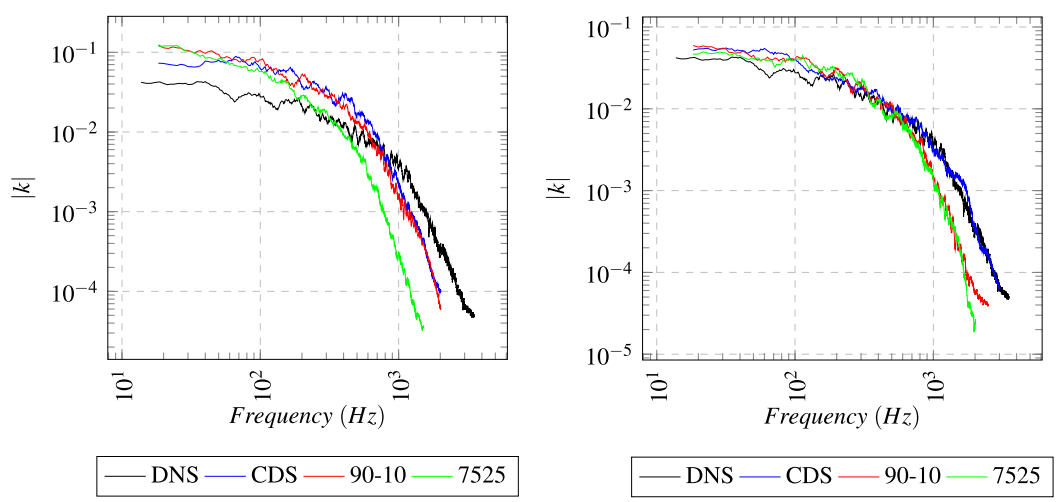

FIG. 29. Spectral plots of the resolved turbulent kinetic energy at the trailing edge for $y^{+}=30$ for meshes $A$ and $\mathrm{C}$. a function of mesh resolution and discretization scheme is shown in Fig. 29. A clear convergence can be seen as the mesh is refined, particularly when the pure central scheme is considered. Interestingly, the overprediction of $\mathrm{k}$ at low wavenumbers is also seen in a recent DNS study, ${ }^{49}$ where simulations of the flow over a NACA0012 are performed at a Reynolds number of 500000 . The aim of the study is to assess the grid convergence. In this study, the frequency spectrum of the turbulent kinetic energy at a point close to the trailing edge is determined and this also shows overpredictions at low wavenumbers for the coarser meshes. This shows that such errors are primarily the result of the discretization process rather than a consequence of the subfilter scale modeling.

The effects of dissipation are also clear in Fig. 29. The higher wavenumber energies are underpredicted when coarsening the mesh or increasing the amount of blending in the discretization scheme.

It is noted that despite large differences in the transition process, the levels of turbulent kinetic energy are not that dissimilar when comparing the different simulations. This is partly due to the fact that spectral information about the transition is lost once the boundary layer becomes fully turbulent. Thus, in terms of the trailing edge flow, the actual mechanism and spectral content of the transition is only important if the boundary layer is still transitional at the trailing edge.

\section{CONCLUSIONS}

DNS and LES have been carried out on the flow over a foil at a moderate Reynolds number to better understand how the large eddy simulations can be used to model flows involving boundarylayer transition. The direct numerical simulations reveal detailed information about the process of the boundary layer transition. The growth of a streamwise disturbance in the separated flow is observed, followed by a secondary three-dimensional instability. The transition process leads to a broadband trailing edge flow, with the initial narrowband component seen in the separation bubble no longer present.

A thorough comparison of the LES and DNS results has been carried out in order to better understand the behavior of LES for transitional boundary layer flows over foils. Direct numerical simulations have provided an excellent set of data against which the large eddy simulations can be compared. Grid independence of the DNS data has been demonstrated, providing a good level of confidence in the data.

The comparison of results from the two SFS models has determined that the Smagorinsky model is unsuitable for capturing the natural transition of the boundary layer. It is well known that this model can be overly dissipative but it is used for studies of boundary layer flows where no external perturbation is applied to the boundary layer. The analysis here shows that the overprediction of the subfilter scale viscosity in the boundary layer prevents transition leading to a laminar boundary layer producing tonal fluctuations at the trailing edge. Grid convergence toward the DNS data is not seen for the three meshes used, indicating that the mesh will need to be at least as refined as those used for the DNS in order to accurately predict the boundary layer flow. It may be the case that transition can be captured if sufficient levels of freestream turbulence or surface roughness are included, but this may result in a different transition mechanism and so the flow would differ from that predicted by the DNS.

From this, we can conclude that the choice of SFS model is a key consideration for modeling transitional boundary layer flows. In particular, an important criterion is that the model remains inactive during the initial stages of transition to allow for the small instabilities to develop. Only when the nonlinear stages of the transition have started should the model become active, which coincides with the emergence of the dissipative turbulent scales. Such behavior was observed for the dynamic $\mathrm{k}$ model, suggesting that it is a suitable model for such flows.

For the dynamic k model, convergence of the results toward the DNS data was seen when considering the mean flow properties, turbulent kinetic energy in the boundary layer, and the spectral content of the flow at the trailing edge. However, none of these simulations fully captured the tonal behavior of the initial streamwise disturbance, which leads to transition in the DNS cases. It is concluded that a mesh resolution commensurate with a DNS would be required to capture the receptive stages of the transition process.

It is interesting to note that despite not accurately capturing the early stages of transition, the large eddy simulations were capable of accurately predicting the boundary layer dynamics once the transition was complete. This implies that it is more important to capture the correct location of the transition rather than the exact mechanism. The spectral content of the transitional flow is lost once the boundary layer becomes fully turbulent and so failing to capture 
this precisely is of less importance. This is only true for boundary layers where the transition is completed well upstream of the trailing edge. Had the process started closer to the trailing edge, then the tonal component would still be present and so capturing this would be important for acoustic predictions. This implies that very high resolutions would be required to capture tonal noise generation from amplified pressure-side Tollmien-Schlichting waves which occur very close to the trailing edge.

The complex interactions between the errors have been explored showing that dispersion errors can cause the natural transition to be bypassed at an earlier stage. The use of blending was shown to reduce the dispersion errors, but this in turn leads to larger dissipation at higher wavenumbers. Despite the error introduced by the blending, it did not introduce so much dissipation as to prevent or delay the transition, and a better convergence toward the DNS data was seen when using this approach. In particular, it has been shown that a small level of up-winding does not introduce so much dissipation so as to replace the SFS model. For simulations where highly refined meshes cannot be used due to complex geometries or high Reynolds numbers, this provides an effective way of minimizing the overall error.

Regarding the verification of large eddy simulations of transitional or turbulent boundary layer flows, this study has shown the importance of looking beyond mean flow properties when undertaking uncertainty analysis. Such analysis should consider quantities such as rms turbulent kinetic energy to understand how the turbulence in the boundary layer changes with mesh resolution. The change in the spectral content of the velocity fluctuations as the mesh is refined has also been shown to be a useful way of identifying errors, which might otherwise not be revealed. The importance of comparing results from multiple grids has also been highlighted. While grid convergence may not always be achievable, comparing results from multiple grids is a highly effective way of identifying different error sources, thus improving confidence in the reliability of a simulation.

\section{ACKNOWLEDGMENTS}

This research was sponsored by the UK Ministry of Defence and by BMT Defence and Security Ltd. The authors also wish to acknowledge the use of the UCL Grace High Performance Computing Facility and associated support services in the completion of this work.

\section{REFERENCES}

${ }^{1}$ H. Schlichting and K. Gersten, Boundary Layer Theory (Springer, 2016).

${ }^{2}$ P. G. Baines, S. J. Majumdar, and H. Mitsudera, "The mechanics of the TollmienSchlichting wave," J. Fluid Mech. 312, 107-124 (1996).

${ }^{3}$ L. E. Jones, R. D. Sandberg, and N. D. Sandham, "Direct numerical simulations of forced and unforced separation bubbles on an airfoil at incidence," J. Fluid Mech. 602, 175-207 (2008)

${ }^{4}$ H. Shan, L. Jiang, and C. Liu, "Direct numerical simulation of flow separation around a NACA 0012 airfoil," Comput. Fluids 34, 1096-1114 (2005).

${ }^{5} \mathrm{~N}$. Thomareis and G. Papadakis, "Effect of trailing edge shape on the separated flow characteristics around an airfoil at low Reynolds number: A numerical study," Phys. Fluids 29, 014101 (2017).

${ }^{6}$ Y. Hoarau, M. Braza, Y. Ventikos, and D. Faghani, "First stages of the transition to turbulence and control in the incompressible detached flow around a NACA0012 wing," Int. J. Heat Fluid Flow 27, 878-886 (2006).
${ }^{7}$ A. Lambert and S. Yarusevych, "Effect of angle of attack on vortex dynamics in laminar separation bubbles," Phys. Fluids 31, 064105 (2019).

${ }^{8} \mathrm{~J}$. W. Kurelek, S. Yarusevych, and M. Kotsonis, "Vortex merging in a laminar separation bubble under natural and forced conditions," Phys. Rev. Fluids 4, 063903 (2019).

${ }^{9}$ L. Brandt, P. Schlatter, and D. S. Henningson, "Transition in boundary layers subject to free-stream turbulence,” J. Fluid Mech. 517, 167-198 (2004).

${ }^{10} \mathrm{~V}$. Ovchinnikov, M. M. Choudhari, and U. Piomelli, "Numerical simulations of boundary-layer bypass transition due to high-amplitude free-stream turbulence," J. Fluid Mech. 613, 135-169 (2008).

${ }^{11}$ S. Lardeau, M. Leschziner, and T. Zaki, "Large eddy simulation of transitional separated flow over a flat plate and a compressor blade," Flow, Turbul. Combust. 88, 19-44 (2012).

${ }^{12}$ T. Sayadi and P. Moin, "Large eddy simulation of controlled transition to turbulence," Phys. Fluids 24, 114103 (2012).

${ }^{13}$ S. Jee, J. Joo, and R.-S. Lin, "Toward cost-effective boundary layer transition computations with large-eddy simulation,” J. Fluids Eng. 140, 111201 (2018).

${ }^{14}$ X. Huai, R. D. Joslin, and U. Piomelli, "Large-eddy simulation of transition to turbulence in boundary layers," Theor. Comput. Fluid Dyn. 9, 149-163 (1997).

${ }^{15}$ S. K. Roberts and M. I. Yaras, "Large-eddy simulation of transition in a separation bubble,” J. Fluids Eng. 128, 232-238 (2006).

${ }^{16}$ E. C. Nash, M. V. Lowson, and A. McAlpine, "Boundary-layer instability noise on aerofoils," J. Fluid Mech. 382, 27-61 (1999).

${ }^{17}$ T. P. Chong, P. F. Joseph, and M. J. Kingan, “An investigation of airfoil tonal noise at different Reynolds numbers and angles of attack," Appl. Acoust. 74, 38-48 (2013).

${ }^{18}$ L. E. Jones and R. D. Sandberg, "Numerical analysis of tonal airfoil self-noise and acoustic feedback-loops," J. Sound. Vib. 330, 6137-6152 (2011).

${ }^{19} \mathrm{~N}$. Curle, "The influence of solid boundaries upon aerodynamic sound," Proc. R. Soc. London, Ser. A 231, 505-514 (1955).

${ }^{20}$ J. E. Ffowcs Williams and D. L. Hawkings, "Sound generation by turbulence and surfaces in arbitrary motion," Philos. Trans. R. Soc., A 264, 321-342 (1969).

${ }^{21}$ I. Solís-Gallego, A. Meana-Fernández, J. F. Oro, K. A. Díaz, and S. VelardeSuárez, "Les-based numerical prediction of the trailing edge noise in a small wind turbine airfoil at different angles of attack," Renewable Energy 120, 241-254 (2018).

${ }^{22}$ H.-J. Kim, S. Lee, and N. Fujisawa, "Computation of unsteady flow and aerodynamic noise of NACA0018 airfoil using large-eddy simulation,” Int. J. Heat Fluid Flow 27, 229-242 (2006).

${ }^{23}$ J. Wang, C. Zhang, Z. Wu, J. Wharton, and L. Ren, "Numerical study on reduction of aerodynamic noise around an airfoil with biomimetic structures," J. Sound. Vib. 394, 46-58 (2017).

${ }^{24}$ W. Zhang, Z. Zou, L. Qi, J. Ye, and L. Wang, "Effects of freestream turbulence on separated boundary layer in a low-Re high-lift LP turbine blade," Comput. Fluids 109, 1-12 (2015).

${ }^{25}$ O. Lehmkuhl, I. Rodríguez, A. Baez, A. Oliva, and C. Pérez-Segarra, "On the large-eddy simulations for the flow around aerodynamic profiles using unstructured grids," Comput. Fluids 84, 176-189 (2013).

${ }^{26}$ L. Davidson, “Large eddy simulations: How to evaluate resolution,” Int. J. Heat Fluid Flow 30, 1016-1025 (2009).

${ }^{27}$ B. J. Geurts and J. Fröhlich, "A framework for predicting accuracy limitations in large-eddy simulation,” Phys. Fluids 14, L41-L44 (2002).

${ }^{28}$ B. J. Geurts, "Analysis of errors occurring in large eddy simulation," Proc. R. Soc. London, Ser. A 367, 2873-2883 (2009).

${ }^{29}$ S. Ghosal, "An analysis of numerical errors in large-eddy simulations of turbulence,” J. Comput. Phys. 125, 187-206 (1996).

${ }^{30}$ D. J. Garmann and M. R. Visbal, "Analysis of tip vortex near-wake evolution for stationary and oscillating wings," AIAA J. 55, 2686-2702 (2017).

${ }^{31}$ L. Jiang, J. Cai, and C. Liu, "Large-eddy simulation of wing tip vortex in the near field,” Int. J. Comput. Fluid Dyn. 22, 289-330 (2008).

${ }^{32} \mathrm{~B}$. Van Leer, "Towards the ultimate conservative difference scheme. V. A second-order sequel to Godunov's method," J. Comput. Phys. 32, 101-136 (1979). 
${ }^{33}$ B. P. Leonard, "The ultimate conservative difference scheme applied to unsteady one-dimensional advection," Comput. Methods Appl. Mech. Eng. 88, 17-74 (1991).

${ }^{34}$ H. Jasak, H. G. Weller, and A. D. Gosman, "High resolution NVD differencing scheme for arbitrarily unstructured meshes," Int. J. Numer. Methods Fluids 31, 431-449 (1999).

${ }^{35}$ S. B. Pope, Turbulent Flows (Cambridge, 2001).

${ }^{36} \mathrm{~S}$. Ghosal and P. Moin, "The basic equations for the large eddy simulation of turbulent flows in complex geometry," J. Comput. Phys. 118, 24-37 (1995).

${ }^{37}$ J. Smagorinsky, "General circulation experiments with the primitive equations: I. The basic experiment," Mon. Weather Rev. 91, 99-164 (1963).

${ }^{38}$ P. Moin and J. Kim, "Numerical investigation of turbulent channel flow," J. Fluid Mech. 118, 341-377 (1982).

${ }^{39}$ E. R. Van Driest, "On turbulent flow near a wall," J. Aeronaut. Sci. 23, 1007-1011 (1956).

${ }^{40}$ U. Piomelli, W. H. Cabot, P. Moin, and S. Lee, "Subgrid-scale backscatter in turbulent and transitional flows," Phys. Fluids A 3, 1766-1771 (1991).

${ }^{41}$ U. Piomelli, T. A. Zang, C. G. Speziale, and M. Y. Hussaini, "On the largeeddy simulation of transitional wall-bounded flows," Phys. Fluids A 2, 257-265 (1990).
${ }^{42}$ W.-W. Kim and S. Menon, "A new dynamic one-equation subgrid-scale model for large eddy simulations," in 33rd Aerospace Sciences Meeting and Exhibit (AIAA, 1995), p. 356

${ }^{43}$ W.-W. Kim and S. Menon, "An unsteady incompressible Navier-Stokes solver for large eddy simulation of turbulent flows," Int. J. Numer. Methods Fluids $\mathbf{3 1}$, 983-1017 (1999).

${ }^{44}$ M. Germano, U. Piomelli, P. Moin, and W. H. Cabot, "A dynamic subgrid-scale eddy viscosity model,” Phys. Fluids A 3, 1760-1765 (1991).

${ }^{45}$ F. K. Chow and P. Moin, "A further study of numerical errors in large-eddy simulations," J. Comput. Phys. 184, 366-380 (2003).

${ }^{46} \mathrm{H}$. K. Versteeg and W. Malalasekera, An Introduction to Computational Fluid Dynamics: The Finite Volume Method (Pearson, 2007).

${ }^{47}$ I. Rodríguez, O. Lehmkuhl, R. Borrell, and A. Oliva, "Direct numerical simulation of a NACA0012 in full stall," Int. J. Heat Fluid Flow 43, 194-203 (2013).

${ }^{48}$ J. C. Hunt, A. A. Wray, and P. Moin, Eddies, Streams, and Convergence Zones in Turbulent Flows (Centre for Turbulence Research, 1988).

${ }^{49}$ M. Gageik, I. Klioutchnikov, and H. Olivier, "Comprehensive mesh study for a direct numerical simulation of the transonic flow at $R e_{c}=500,000$ around a NACA 0012 airfoil," Comput. Fluids 122, 153-164 (2015). 